\title{
单质碘促进的有机反应研究进展
}

\author{
赵巾巾高文超* 常宏宏* 李兴刘强魏文珑 \\ (太原理工大学化学化工学院生物与制药工程系 太原 030024)
}

\begin{abstract}
摘要 单质碘作为有机化学中结构最为简单的反应试剂之一, 其促进的有机转化在最近几年越来越受到人们的关注. 总结了单质碘的氧化性, 酸性和亲电性 3 类基本化学性质, 并针对 3 类性质所涉及的相关反应性报道进行了介绍. 除此 之外, 还总结了近 5 年来单质碘作为催化剂在杂环合成, 偶联反应, 及 $\mathrm{C}-\mathrm{H}$ 官能团转化等方面的应用.
\end{abstract}

关键词 单质碘; 氧化; 酸性; 亲电环化; 杂环合成; 有机催化

\section{Recent Advances in lodine-Mediated Organic Reactions}

\author{
Zhao, Jinjin Gao, Wenchao* Chang, Honghong* \\ Li, Xing Liu, Qiang Wei, Wenlong \\ (College of Chemistry and Chemical Engineering, Taiyuan University of Technology, Taiyuan 030024)
}

\begin{abstract}
As one of simplest chemical reagents, iodine has promoted diverse organic transformations, which have attracted much attention in recent years. The present review summarizes three basic chemical features of iodine in organic synthesis: the oxidative reactivity, the acidic reactivity and the electrophilic reactivity, along with the introduction of corresponding representative examples. Furthermore, the iodine-catalyzed reactions involed in the synthesis of heterocycles, organic coupling reactions, the $\mathrm{C}-\mathrm{H}$ functionalization, and other applications are also presented in this review.

Keywords iodine; oxidation; acidity; electrophilic cyclisation; heterocycle syntheses; organocatalysis
\end{abstract}

碘是一种卤族元素, 原子序数为 53 , 位于元素周期 表的第 5 周期, 第 VIIA 族, 属 $\mathrm{p}$ 区元素. 碘元素是由法 国科学家库尔图瓦利(Bernard Courtois)于 1811 年在海 藻灰的提取液中首次发现 ${ }^{[1]}$. 碘元素虽然在自然界丰度 不高(丰度在地壳元素中排 47 位), 但分布十分广泛, 普 遍存在于无机矿石和有机生物体内. 目前世界上所使用 的碘元素主要有两个来源, 一种来源于海水(在海洋矿 井或油田附近开采出的富含碘元素无机盐, 如日本的南 关东天然气田附近的探明的无机盐中碘元素的含量在 $100 \mathrm{mg} / \mathrm{kg}$ 以上), 二是来源于智利的含有碘酸盐的矿石 $\left[\mathrm{Ca}\left(\mathrm{IO}_{3}\right)_{2}\right.$ 和 $\left.\mathrm{Ca}\left(\mathrm{IO}_{3}\right)_{2} \cdot 8 \mathrm{CaCrO}{ }_{4}\right]$. 海水中分离出的离子态 碘的无机盐可以通过氧化剂(如氯气)的氧化得到游离态 的磑, 后者则通过加入亚硫酸氢钠将含有碘酸盐的硝石 还原而得到.

目前世界上对碘元素的需求大概分成以下几方面
(图 1): X 射线造影剂(21\%), 消毒剂和杀菌剂(20\%), 有 机化学中的反应试剂(19\%)，制药业及药物中间体 $(16 \%)$, 动物饲料 $(9 \%)$, 除草剂 (4\%)，摄影成像材料 $(3 \%)$, 其他应用 $(8 \%)^{[2]}$. 本文所讨论的内容主要集中于 碘元素在有机反应领域中的应用.

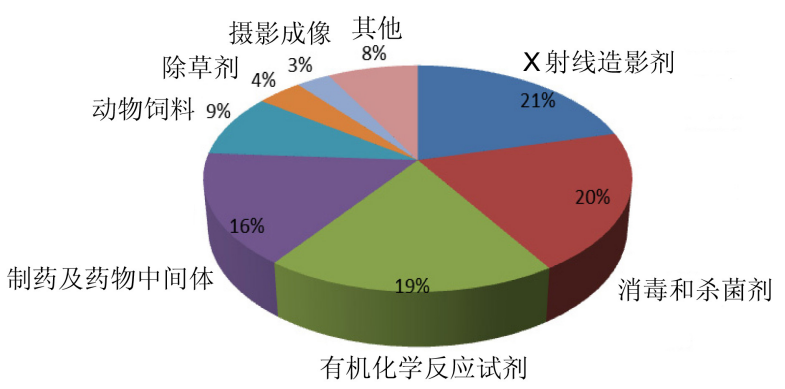

图 1 单质碘的需求分布图

Figure 1 The world demand for iodine or organic iodine compounds

\footnotetext{
*E-mail: gaowenchao@tyut.edu.cn; changhonghong@tyut.edu.cn

Received May 4, 2014; revised June 2, 2014; published online June 23, 2014.

Project supported by the Natural Science Foundation of Shanxi Province (Nos. 2012021007-2, 2011011010-2), the Scientific and Technologial Innovation Programs of Higher Education Institutions in Shanxi Province (No. 20120006), and the Qualified Personnel Foundation of Taiyuan University of Technology (No. tyut-rc201307a).

山西省自然科学基金(Nos. 2012021007-2, 2011011010-2)、山西省高等学校科技创新基金(No. 20120006)、太原理工大学引进人才启动经费(No. tyut-rc201307a)资助项目.
} 
单质碘是双原子分子, 常温常压下为紫黑色有光泽 的固体, 熔点 $113.5{ }^{\circ} \mathrm{C}$, 沸点 $184.4{ }^{\circ} \mathrm{C}$, 密度为 4.93 $\mathrm{g} / \mathrm{m}^{3}\left(2{ }^{\circ}{ }^{\circ} \mathrm{C}\right)$. 由于单质碘 $\mathrm{I}-\mathrm{I}$ 键的键长为 $270 \mathrm{pm}$, 是 目前已知的最长的单键之一, 导致了 I-I 键能较弱 $\left(\Delta H_{298}=150.7 \mathrm{~kJ} / \mathrm{mol}\right)^{[3]}$, 受到极化作用时极易发生断 裂. 固态单质碘加热时会直接转变为气态, 形成有毒的 紫红色碘蒸气, 这即是所谓的升华现象. 单质碘在许多 有机溶剂中都有很好的溶解性, 但在水中的溶解性较 差. 然而如果水溶液中有 $\mathrm{I}^{-}$离子存在, 单质碘会与其形 成 $I_{3}^{-}$, 使得溶解性大大提高. 除此之外, $\mathrm{I}^{-}$与 $\mathrm{I}_{2}$ 相互结 合还可以形成一系列的多磑化物的聚集态(从 $\mathrm{I}^{-}$到 $\mathrm{I}_{29}^{3-}$ ), 这些不同聚集态的碘化物具有不同的导电性能, 在导电 材料, 太阳能电池等领域有重要应用 ${ }^{[4]}$.

碘元素是人体内不可缺少的微量元素, 尤其在调节 人体生长发育过程中发挥着重要作用, 健康成人体内的 碘元素总含量为 $20 \sim 50 \mathrm{mg}$. 目前, 已知碘元素在人体 内的唯一生理角色是在甲状腺(thyroid gland)中合成甲 状腺激素(thyroid hormones). 碘元素从饮食中摄入后通 过胃和小肠吸收, 吸收后各种形式的碘元素在人体内都 转化成负一价离子态的碘, 食物和水中摄取的负一价碘 离子可以 $100 \%$ 被人体吸收并利用. 碘离子被吸收后进 入血液循环系统, 并在甲状腺中聚集合成甲状腺素(甲 状腺激素的主要成分), 碘元素的所有生物学表现都是 围绕甲状腺素进行的. 被人体利用的碘元素主要以三碘 甲状腺原氨酸 T3 (Triiodothyronine) 和四碘甲状腺原氨 酸 T4 (Tetraiodothyronine) (Scheme 1)的形式储存在甲状 腺球蛋白中, 这是甲状腺素的活性形式, 它可以与细胞 核上的受体结合后控制一类特殊基因的表达, 进而调节 人体的新陈代谢 ${ }^{[5]}$.<smiles>N[C@@H](Cc1cc(I)c(Oc2ccc(O)c(I)c2)c(I)c1)C(=O)O</smiles>

Triiodothyronine<smiles>N[C@@H](Cc1cc(I)c(Oc2cc(I)c(O)c(I)c2)c(I)c1)C(=O)O</smiles>

Scheme 1

单质碘作为有机化学领域结构简单, 性质稳定的试 剂之一, 由于其廉价易得 $67.00 \$ / \mathrm{kg}$ ), 操作简便, 反应 丰富等性质在有机合成中有着广泛的应用，除了最常见 的磑化反应(芳环上的取代反应、酮 $\alpha$ 位的取代反应、
碘仿反应以及与不饱和键的加成反应等), 单质碘还有 着其他多种多样的反应性. 尽管已有许多综述对其反应 性作了一定的概括和总结，然而这些综述性文章大多集 中于单一反应性的概括(如亲电碘环化反应 ${ }^{[6]}$ 、 Lewis 酸

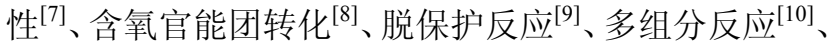
氧化芳香化反应 ${ }^{[11]}$ 等)，对单质碘产生相关反应性的原 因及不同反应性之间的相关性涉及不多; 而有些综述在 总结单质碘的反应性的同时，对许多其他价态碘元素的 反应性(如碘负离子的反应性 ${ }^{[2 b]}$ 、高价碘的反应性 ${ }^{[12]}$ 等) 也多有涉及，而这些反应性已不属于单质碘的反应性范 畴. 本文在前人相关综述的基础上，专一性地对单质碘 的基本反应性作了系统的概括，根据单质碘的结构特征 阐述了相关性质产生的原因, 并且对近 5 年来所发表的 单质碘催化的有机转化做了详细的分析.

\section{1 氧化性}

单质碘属于一种弱氧化剂, 其发挥氧化性的原因主 要有以下 3 点: (1)在有机碱或者无机碱的存在下, 单质 碘可以发生歧化, 生成次碘酸离子 $\left(\mathrm{IO}^{-}\right)$、亚碘酸离子 $\left(\mathrm{IO}_{2}^{-}\right)$、或者碘酸根离子 $\left(\mathrm{IO}_{3}^{-}\right)$, 正是由于溶液中多种多 样活性更高的碘离子中间体的存在，共同作用完成了氧 化转化; (2)由于碘元素在非放射性卤族元素中具有最低 的均裂能, 在光照或者加热的条件下很容易将其解离形 成自由基，从而可以完成许多单电子转移的氧化反应; (3)除了放射性卤族元素砹之外, 碘元素电负性最小, 因 此，与其他卤族元素 $(\mathrm{F}, \mathrm{Cl}, \mathrm{Br})$ 或者极性官能团相互作 用时单质碘更容易受到诱导形成 “ $\mathrm{I}^{+}$” , 进而起到氧化 作用 ${ }^{[2 b]}$.

\section{1 醇或醛的氧化}

在相应的亲核试剂存在下，单质碘可以将醇或者醛 氧化成酯、酰胺或者嫝类化合物. 例如, 以甲醇为溶剂, 将脂肪醛用 $\mathrm{I}_{2}$ 和 $\mathrm{KOH}$ 处理, 可以以几乎定量的收率得 到相应的甲酯(Scheme 2). 通常认为, 此反应首先由醛 与甲醇反应生成对应的半缩醛，半缩醛被碘正离子(或 者次碘酸离子)氧化，接着在碱的作用下消除碘化氢而 得到最终酯类产物 ${ }^{[13]}$.

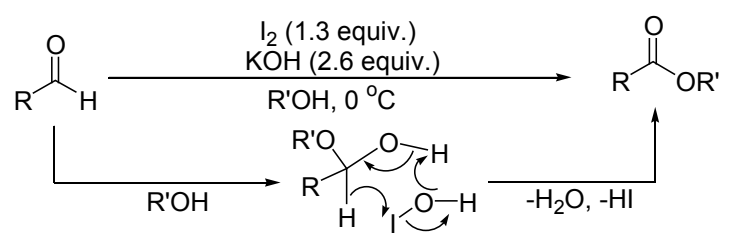

Scheme 2

另一个较为典型的例子是将醇氧化成相应的腈. 在 单质碘的存在下，以 $28 \%$ 氨水溶液为溶剂, 伯醇可以 60 
${ }^{\circ} \mathrm{C}$ 条件下被氧化为对应的氰基化合物(Scheme 3), 反应 历程涉及到两个对应的氧化中间体 C- $\mathrm{OI}$ 和 $\mathrm{C}=\mathrm{NI}$, 最 终消除碘化氢得到腈类产品 ${ }^{[14]}$.

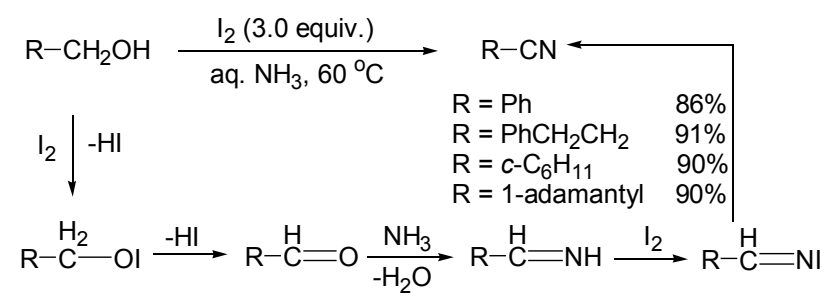

Scheme 3

\section{2 硫醚(酚)氧化}

单质碘氧化含硫化合物的应用之一, 就是在杀菌消 毒过程中, 将病毒或者细菌蛋白质结构中半胱氨酸的颈 基氧化成二硫化合物, 致使蛋白质变性而消灭这些致病 微生物 ${ }^{[2]}$. 在有机合成中, 人们也经常利用单质碘的这 个特性将硫醇或者硫酚转化成二硫化合物 ${ }^{[15]}$. 对于硫 醚, 在水的存在下, 单质碘可以将其氧化成亚砜 (Scheme 4) ${ }^{[16]}$.

$$
R^{1-S} R^{2}+I_{2} \rightleftharpoons R^{1-S_{I^{-}}^{+}} R^{2} \frac{H_{2} \mathrm{O}}{-H I} R^{1-S_{\backslash}} R^{2}
$$

\section{Scheme 4}

\section{3 氧化形成 C-C 键}

富电子芳环极易在光照下被单质碘氧化产生活性 自由基, 又由于邻二芳基苯的 $\mathrm{p}$ 轨道在空间上的相互接 近, 从而可以发生自由基偶联形成 $\mathrm{C}-\mathrm{C}$ 键(Eq. 1). 反 应需用 $400 \mathrm{M}$ 高压录灯照射, 并且要过量的单质碘参 与 ${ }^{[17]}$. 如果将连接两个芳环的碳链延长, 反应不能发 生，这说明空间上的距离对该反应影响很大.

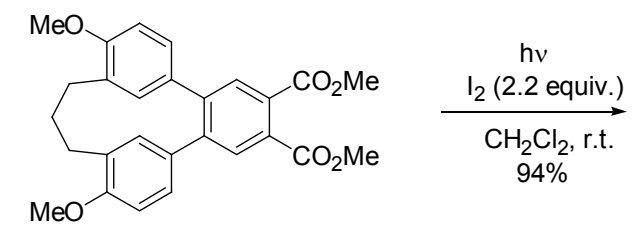<smiles></smiles>

单质碘在甲醇钠的作用下与对溴苯乙腈反应，可以 发生脱氢二聚(Scheme 5). 反应历程依次经过单质碘氧 化形成自由基、自由基偶联、偶联产品碘化、碘化氢的
消除得到最终的产品 ${ }^{[18]}$.

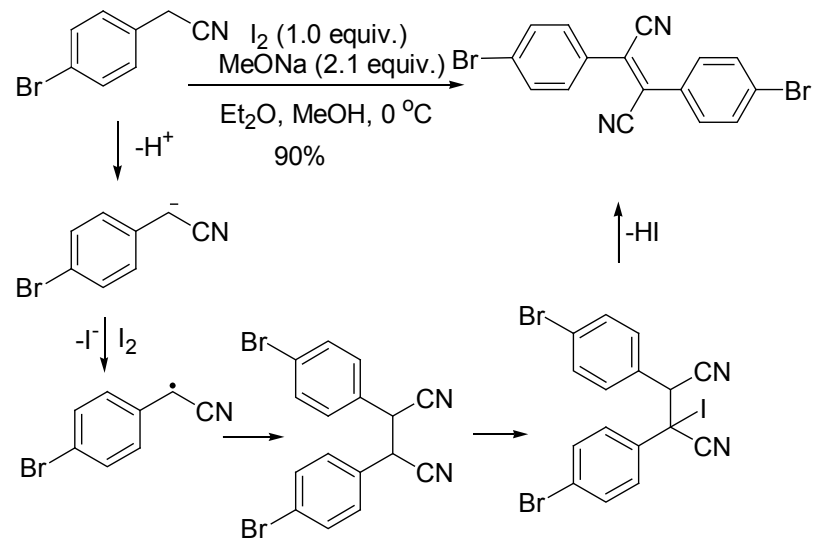

Scheme 5

马大为小组 ${ }^{[19]}$ 以单质碘促进的分子内氧化偶联反 应为关键成环步骤完成了 4 种天然产物的全合成 (Schemes 6 9). 反应需要首先在低温条件下用六甲基 二硅基氨基锂(LiHMDS)处理前体化合物(溶剂一般使用 四氢呋喃(THF)或者六甲基磷酰三胺(HMPA), 吲哚氮 原子和活泼羰基(酰胺或者丙二酸酯) $\alpha$ 位的质子均会被 拔除而形成两个带负电荷的基团, 吲哚部分的负电荷由 于共振作用会部分转移至 3 位的碳原子上, 由于两个碳 负离子之间相隔 4 个原子, 形成 $\mathrm{C}-\mathrm{C}$ 键后会构建一个 热力学最稳定的六元环系, 反应体系中加入单质碘后会 诱导两个碳原子之间发生偶联关环反应，收率都在中等 以上, 并且具有很高的立体专一性, 他们 ${ }^{[19 b]}$ 推测机理 是一个自由基氧化的过程. 这 4 种天然产物的精妙合成, 充分体现了单质碘在氧化偶联形成 $\mathrm{C}-\mathrm{C}$ 键方面的巨大 优势.
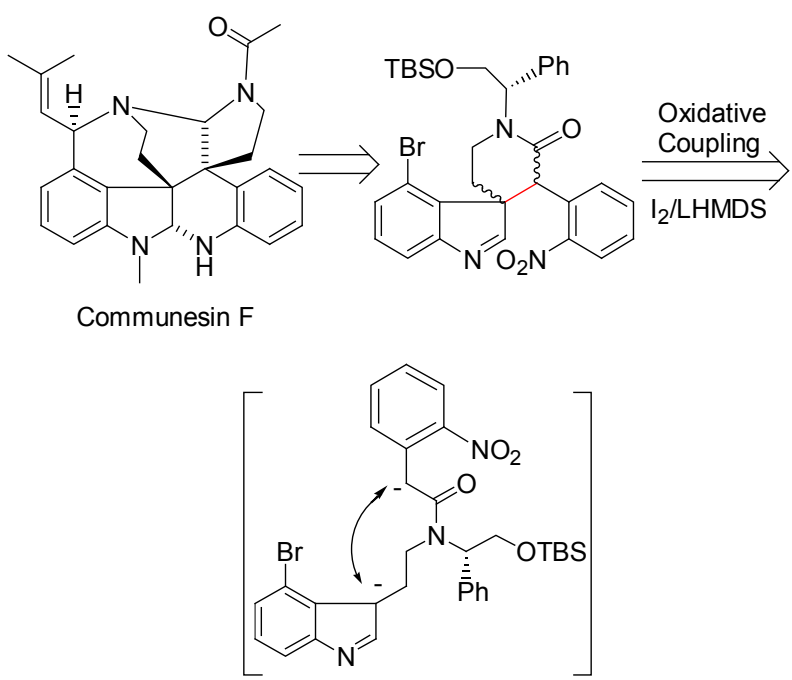

Scheme 6 

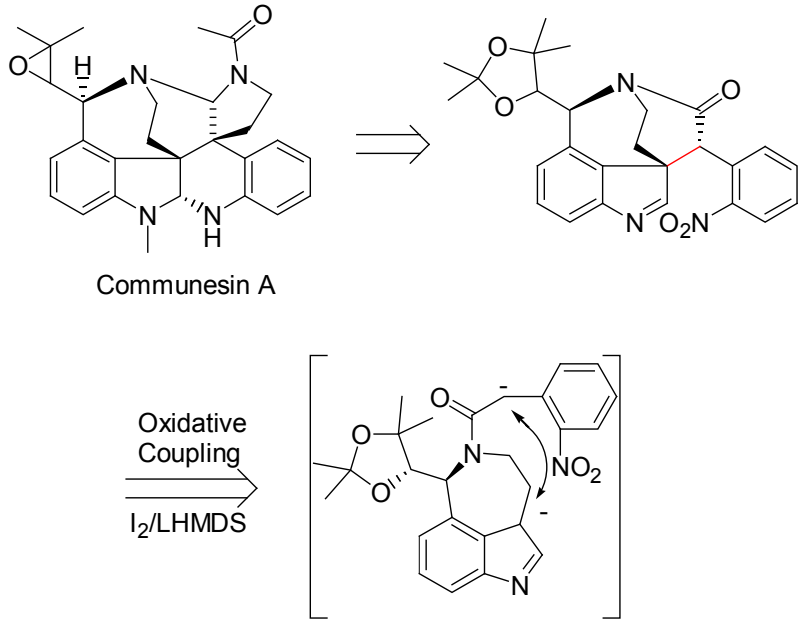

Scheme 7
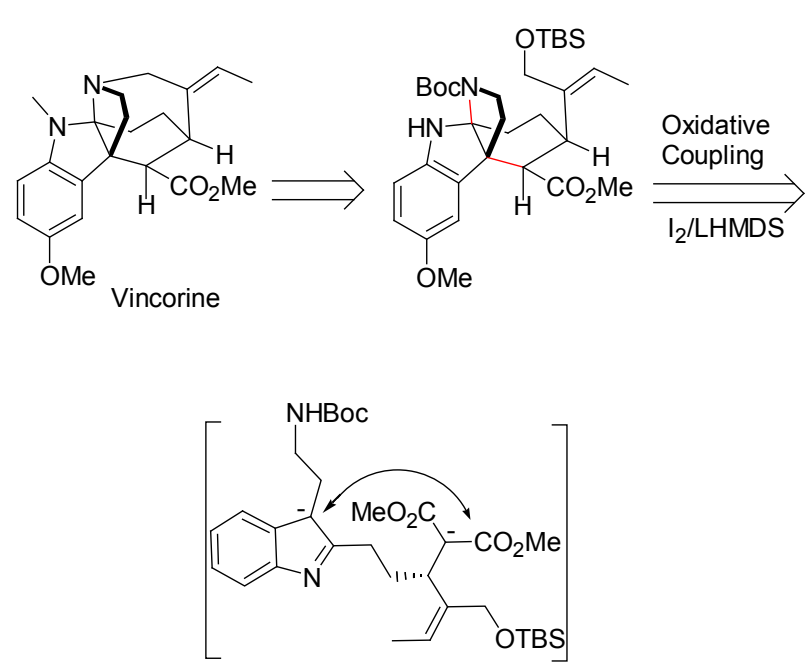

Scheme 8
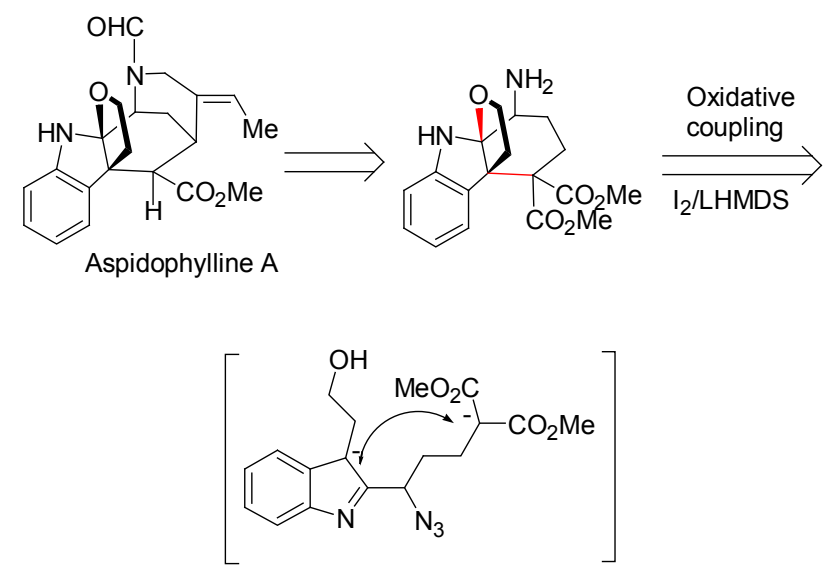

Scheme 9

一级卤代烷烃用二甲基亚砜氧化得到醛类的反应， 叫作 Kornblum 氧化. 吴安心小组 ${ }^{[2]}$ 使用 3 类含氮芳香 化合物一一吲哚、 $N, N$-二甲基苯胺，以及吡啶分别与芳
香甲基酮反应，可以通过分子间 $\mathrm{C}-\mathrm{C}$ 成键反应构筑复 杂的分子结构. 其中涉及到的主要反应是 $\alpha$-碘代甲基酮 的 Kornblum 氧化, 由于 $\alpha$-羰基醛活性很高, 很容易受 到亲核试剂(如吲哚、 $N, N$-二甲基苯胺以及吡啶)的进攻, 所生成的活性中间体可以进一步发生加成, 取代等反应 得到目标化合物(Scheme 10).

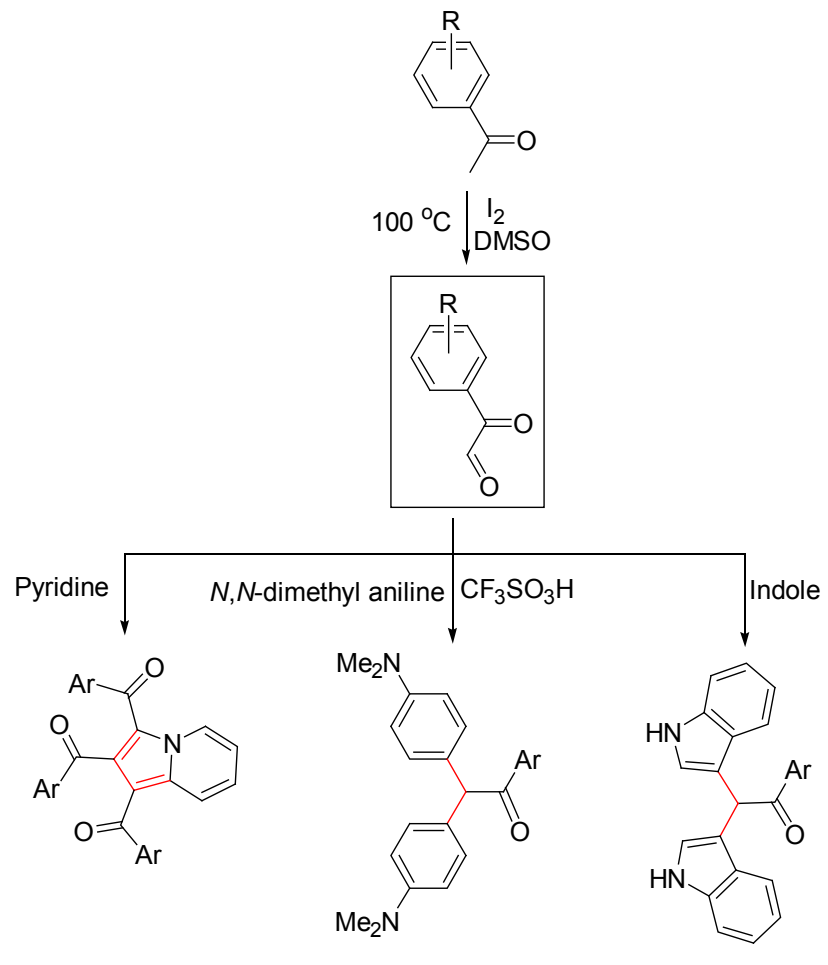

Scheme 10

2-荎酚也是具有较高亲核活性的芳香体系. 利用单 质碘和 DMSO 完成对甲基酮的 Kornblum 氧化之后与 2荟酚反应(Eq. 2)，可以得到菜并呋喃酮的结构。该方法 在完成了 $\mathrm{C}-\mathrm{C}$ 成键的同时, 还构筑了一个具有半缩酮 结构的季碳中心 ${ }^{[21]}$.
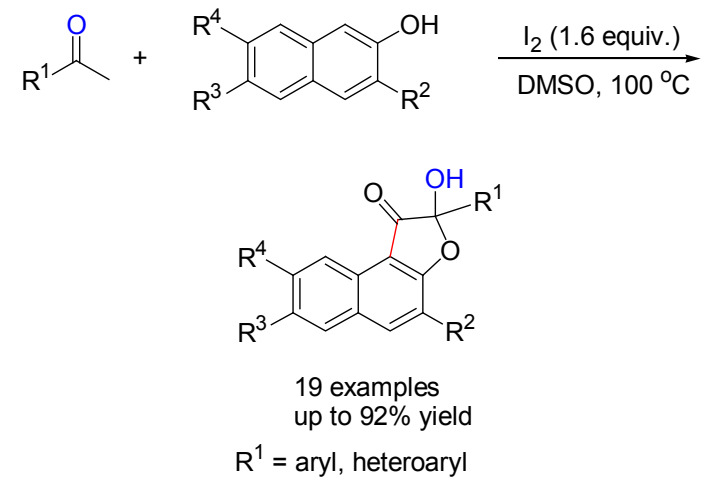

此外，如果反应体系中有氧化铜存在，芳基甲基酮 在单质碘的作用下会发生自身偶联 $\mathrm{C}-\mathrm{C}$ 成键反应，生 成共轭烯酮的结构 (Eq. 3)，同时会将二甲基亚砜 (DMSO)中的硫甲基引入到产物中 ${ }^{[22]}$. 


$$
\begin{aligned}
& \stackrel{\substack{\mathrm{I}_{2} \text { (2 equiv.) } \\
\mathrm{CuO} \text { (3 equiv.) }}}{\mathrm{DMSO}, 65^{\circ} \mathrm{C}} \prod_{\mathrm{O}}^{\mathrm{R}} \\
& \mathrm{R}=\text { Aryl or Heteroaryl }
\end{aligned}
$$

\section{4 氧化形成 $\mathrm{C}-\mathrm{N}$ 键}

第一种单质碘促进的 $\mathrm{C}-\mathrm{N}$ 成键反应是通过甲基酩 发生氧化再与胺类化合物缩合完成的. 2013 年吴安心小 组 ${ }^{[23]}$ 利用单质碘在 DMSO 溶剂中可以促使甲基酮发生 Kornblum 氧化形成活性较高的苯甲酰基甲醛, 邻氨基 苯甲酰胺与苯甲酰基甲醛发生亚胺缩合环化，最终形成 氧化嘧啶的杂环结构 (Scheme 11). 利用该方法该小 组完成了 Luntonin F 及其衍生物的合成.

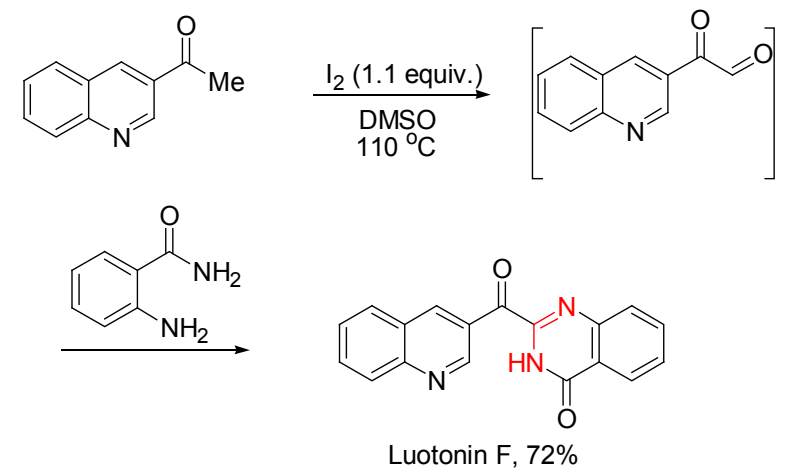

Scheme 11

单质碘在 DMSO 中可以将芳基甲基酮转化成苯甲 酰基甲醛这一转化还可以合成其他杂环结构. 例如, 使 用2-氨基吡啶与原位生成的苯甲酰基甲醛反应，可以得 到吡啶并咪唑的杂芳环结构(Eq. 4), 反应不需要金属试 剂的参与, 可以一次形成 3 个 $\mathrm{C}-\mathrm{N}$ 键 ${ }^{[24]}$.

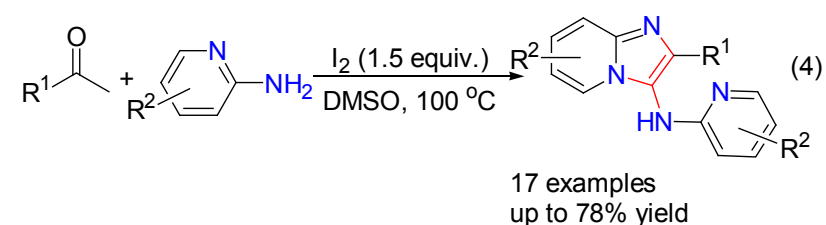

2009 年, 吴安心小组 ${ }^{[25]}$ 使用氨水为溶剂, 可以将甲 基酮或者甲基仲醇在单质碘的作用下转化成酰胺 (Scheme 12). 底物中的甲基酮被氧化生成三碘代甲基 酮, 受到氨分子的进攻后三碘代甲基以碘仿的形式离 去，氨基保留就形成相应的酰胺结构.

另外一种 $\mathrm{C}-\mathrm{N}$ 成键的方式是借助单质碘对活性双 键的活化作用完成的. 吲哚的 2 位并不是一个高活性的 位点, 然而在单质碘的存在下, 通过活化吲哚 2,3 位的 双键, 可以专一性地在吲哚 2 位引入含氮或者含碳基团.

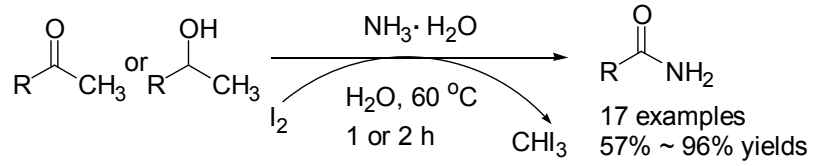

\section{Scheme 12}

梁永民小组 ${ }^{[26]}$ 使用单质碘配合不同的无机碱，可以完 成吲哚 2-位 $\mathrm{C}-\mathrm{N}$ 成键, 分别引入仲胺或者磺酰胺(Eq. $5)$, 同时利用该 $\mathrm{C}-\mathrm{N}$ 成键策略，该小组 ${ }^{[26 b]}$ 完成了天然 产物 $( \pm)$-Folicanthine 的全合成. 黄精美小组 ${ }^{[27]}$ 使用饱 和甲酸铵溶液与 $1,4-$ 二氧六环的混合溶液为溶剂, 不使 用任何有机或者无机碱完成了吲哚 2 位与二唑或者三唑 的 $\mathrm{C}-\mathrm{N}$ 成键反应，条件温和并具有很高的收率和区域 选择性.
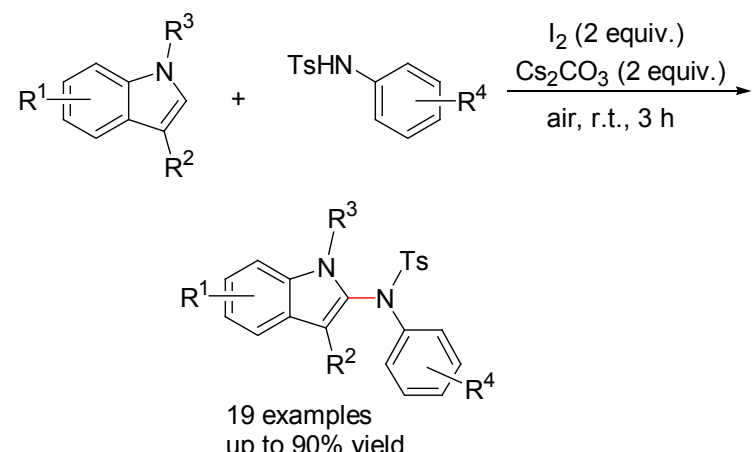

最近，我们 ${ }^{[28]}$ 发展了一种单质碘促进的分子内的 $\mathrm{C}-\mathrm{N}$ 成键反应，通过调控氨基的保护基可以控制反应 生成 2-酰基吲哚或者 2-酰基吲哚啉的产物(Scheme 13). 通过对反应机理的研究, 我们认为反应直接或者间接通 过活化烯醇的双键，从而形成活性较高的 $\alpha$-碘代酮与分 子内的酰胺类官能团反应完成环化过程. 同时，运用该
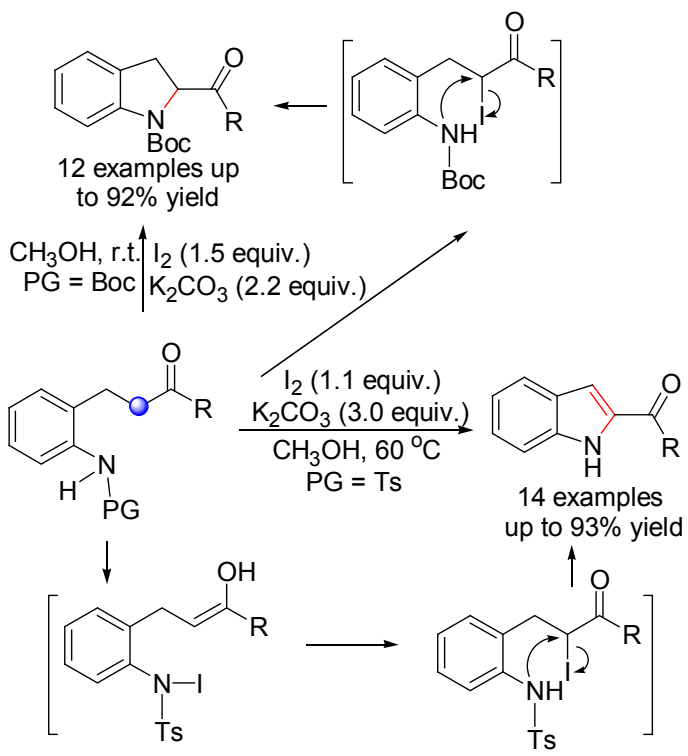

Scheme 13 
方法完成了天然产物 Quindolinone 全合成, 较之以往的 合成方法更为简便高效.

\section{5 氧化形成 $\mathrm{C}$ - $\mathrm{O}$ 键}

单质碘或者碘负离子与有机氧化剂配合使用可以 完成许多 $\mathrm{C}-\mathrm{O}$ 成键的反应 ${ }^{[2 \mathrm{~b}]}$, 然而单独使用单质碘为 氧化剂形成 $\mathrm{C}-\mathrm{O}$ 成键的报道并不多. 2013 年, 郑州大 学常俊标小组 ${ }^{[29]}$ 使用苯甲醛与酰肼缩合形成的亚胺化 合物, 在单质碘的作用下可以完成分子内 $\mathrm{C}-\mathrm{O}$ 成键构 筑噁二唑结构. 单质碘活化亚胺双键可以得到 $\alpha$-碘代偶 氮中间体，中间体中的碳原子受到分子内羰基氧原子的 进攻而形成 $\mathrm{C}-\mathrm{O}$ 键, 从而得到 1,3,4-腎二唑的杂环结 构(Scheme 14). 反应底物范围广，合成效率高.

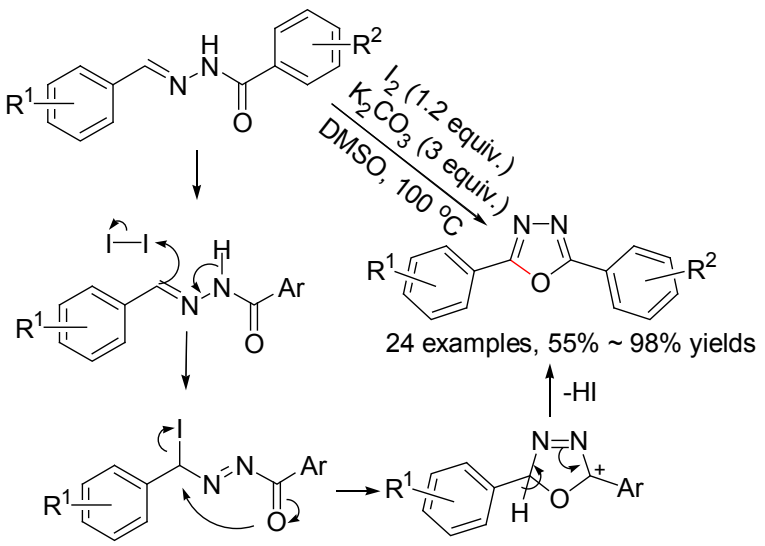

Scheme 14

\section{6 氧化形成 $\mathrm{C}-\mathrm{S}$ 键}

在前面的叙述中我们已经提到，单质碘可以氧化硫 醚形成亚砜, 氧化硫醇形成二硫键 ${ }^{[15,16]}$, 说明硫醇或者 硫醚的抗氧化能力较弱. 因此, 若要实现氧化形成 $\mathrm{C}-$ $\mathrm{S}$ 键的目的, 所使用的底物往往是具有较高氧化态的砜 类或者亚砜类的底物. 在单质碘的作用下，烯烃类底物 可以在端位引入砜基, 而对于炔烃类底物, 则主要生成 $\alpha$-碘代烯基砜, 并且使用苯乙炔的效果要比脂肪炔类反 应效果好 $(\text { Scheme 15 })^{[30]}$.

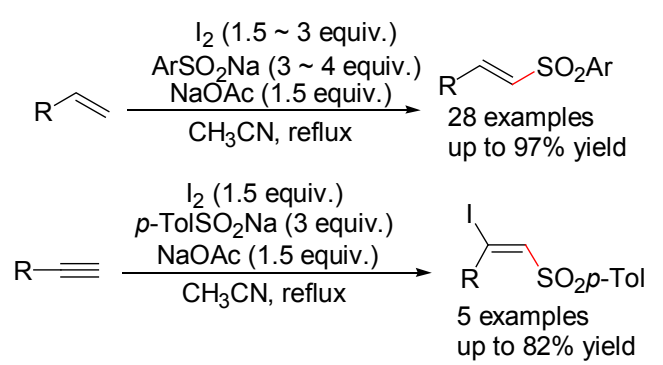

\section{Scheme 15}

当然, 如果使用抗氧化性较强的含硫杂环化合物,
同时结合单质碘在 DMSO 中能够促使甲基酮转化成苯 甲酰基甲醛这一特点，也可以完成 $\mathrm{C}-\mathrm{S}$ 成键反应. 例 如，使用苯丙噻唑和芳基甲基酮为底物，以 DMSO 为溶 剂, 在单质碘和 $\mathrm{KOH}$ 的作用下可以完成 $\mathrm{C}-\mathrm{S}$ 成键以及 $\mathrm{C}-\mathrm{N}$ 成键的双重反应，得到 2-酰基噻唑类化合物(Eq. $6)^{[31]}$.

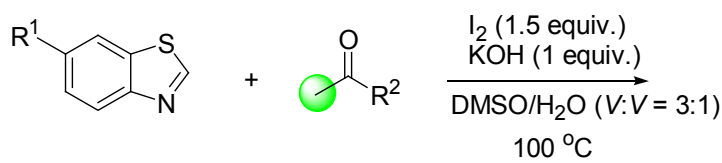<smiles>[R]C(=O)c1nc2ccc([R])cc2s1</smiles>

27 examples up to $95 \%$ yield

\section{7 氧化构筑芳香体系}

氧化脱氢构筑芳香体系是单质碘常用的氧化反应. 具有共轭结构的环己烯酮化合物在甲醇中用单质碘处 理可以得到取代的苯酚，环己烯酮上共轭基团越多，则 反应越容易进行，收率越高(Eq. 7) ${ }^{[32 \mathrm{a}]}$. 例如，在环已烯 酮中 $\gamma$ 位引入酯基，可以将芳香化产品收率提高至 $80 \%$ 以上 ${ }^{[32 b]}$.

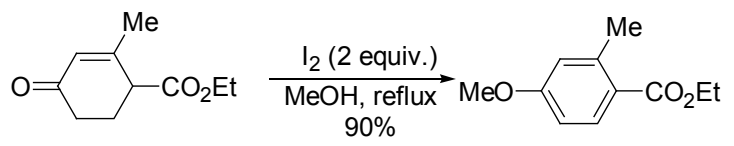

除了全碳环系的芳构化之外，杂原子环系也可以被 氧化形成芳香体系. 2000 年 Yadav 小组 ${ }^{[33]}$ 报道了单质碘 可将 1,4-二氢吡啶在甲醇回流的条件下氧化成吡啶(Eq. 8). 如果反应体系中加入碱(如 $\mathrm{KOH}$ )能加速反应进行.

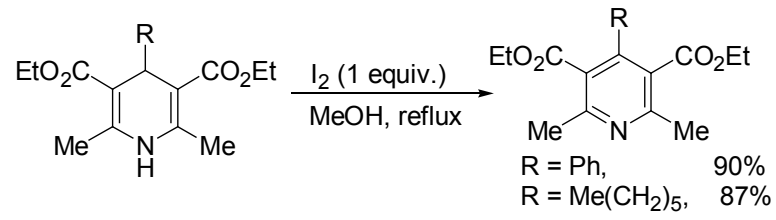

\section{2 酸性}

单质碘作为酸性催化剂在有机反应中有着广泛的 应用，然而其在不同的反应中发挥的作用不同：当碘在 质子性溶剂(如甲醇、乙醇)中加热时，会有部分 HI 生 成 ${ }^{[34]}$, 催化量的 HI 在体系中起 Brønsted 酸的作用; 另一 方面，在非质子性溶剂或者无溶剂条件下，由于碘较大 的原子半径及多电子层结构, 碘的 $\mathrm{d}$ 及 $\mathrm{p}$ 轨道可以与有 机分子中的孤对电子或者共轭体系中的 $\pi$-电子配位，形 成电荷转移中间体 ${ }^{[35]}$, 使得其能发挥 Lewis 酸的作用. 


\subsection{Brønsted 酸催化剂}

以甲醇为溶剂, 脂肪羧酸在单质碘的催化作用下可 以几乎定量地得到甲酯类产物, 而芳香羧酸则不发生反 应(Eq. 9). 由于反应活性存在伯醇 $>$ 仲醇 $>$ 叔醇的规律, 作者认为该反应是单质碘与甲醇反应生成的催化量的 $\mathrm{HI}$, 通过活化醇羟基促进羧基负离子的亲核进攻 ${ }^{[36]}$.

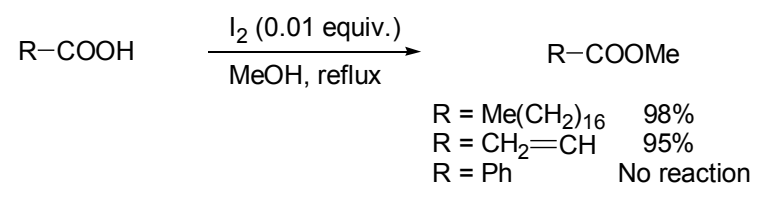

在单质碘促进的含氧官能团的保护及去保护的反 应中, 多数人认为催化量的单质碘与质子性溶剂反应生 成的 HI 起主要作用 ${ }^{[37]}$. 例如, 对于硅基保护基的脱除, 单质碘显示了其优越性. 在苯甲酰基 $(\mathrm{Bz})$ 、茮基 $(\mathrm{Bn})$ 、 甲基(Me)以及缩酮等保护基同时存在时, 可以专一性地 完成硅基保护基(叔丁基二甲基硅基，TBDMS)的脱除， 而对以上三类保护基没有任何影响(Eq. 10) ${ }^{[38]}$.

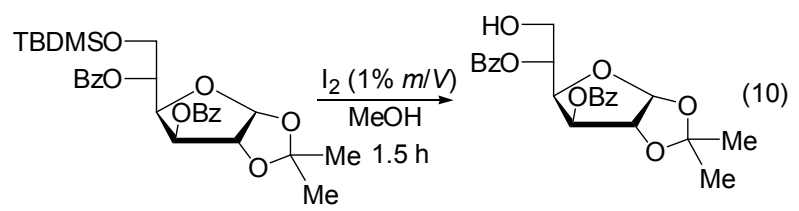

以甲醇为溶剂, 三苯基甲基( $\mathrm{Tr}$ )保护的醇羟基可以 使用催化量的单质碘予以脱除(Eq. 11), 在其他醇类溶 剂也可以完成该转化, 然而使用非质子性溶剂如四氯化 碳等, 即使反应回流 2 周, 保护基仍然不能脱除 ${ }^{[39 a]}$. 该 脱保护的方法已经在天然产物 Sylvaticin 的全合成中得 到验证 $^{[39 b]}$.

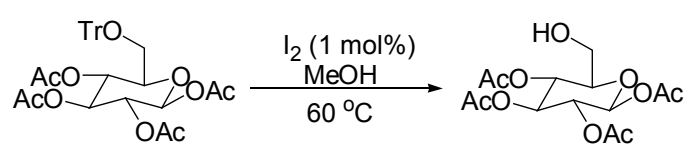

此外, 在质子性溶剂中, 单质碘发挥 Brønsted 酸的 作用在脱除对甲氧基苯基醚保护基，催化合成缩酩、缩 醛等反应中均显示了良好的效果 ${ }^{[37]}$.

\subsection{Lewis 酸催化剂}

单质碘还经常作为 Lewis 酸在有机合成中催化某些 反应. 与其他金属 Lewis 酸相比, 单质碘有三个明显的 优点: 对空气和水不敏感; 价格低廉; 后处理简便 (用 还原剂如 $\mathrm{Na}_{2} \mathrm{~S}_{2} \mathrm{O}_{3}$ 洗涤即可 $)^{[40]}$. 例如在 Aldol 反应 ${ }^{[41 \mathrm{a}]}$ 、 Mannich 反应 ${ }^{[41 \mathrm{~b}, 41 \mathrm{c}]}$ 、Michael 加成反应 ${ }^{[41 \mathrm{~d}]}$ 、PictetSpengler 反应 ${ }^{[41 e, 41 f]}$ 中, 都可以见到许多成功的例子. 通 常情况下, 使用催化量的单质碘活化底物中的羰基或者 亚胺中间体，促进亲核试剂对底物的进攻从而完成反
应。例如在苯硫酚对烯酮的 Michael 加成中，使用 5 $\mathrm{mol} \%$ 的单质碘可使烯酮的羰基被活化，增强了 $\alpha$ 位碳 原子的电正性，使得可以高收率地完成加成反应(Eq. 12). 当然, 对于有些多组分反应，使用催化量的单质碘 反应速率较慢，增加碘的用量对反应转化率及提高反应 效率也是有利的(Eqs. 12, 13).<smiles>[R]C=CC([R])=O</smiles><smiles>[R16][CH+]C([R])=O</smiles>

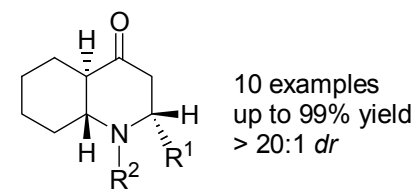

王彦广小组 ${ }^{[42]}$ 使用单质碘为催化剂, 以亚胺和醛 为底物, 可以高效地合成喹啉类杂环化合物 (Scheme 16). 在该反应中, 单质碘作为 Lewis 酸催化剂, 主要完 成了两个过程，一个是醛与亚胺的 Aldol 反应，另一个 是分子内的傅克环化反应.

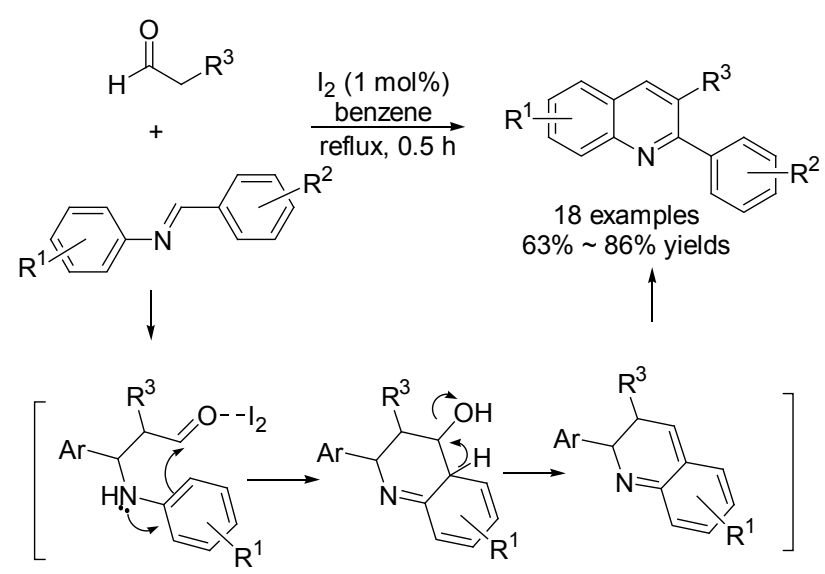

\section{Scheme 16}

2004 年 Loh 小组 ${ }^{[43]}$ 报道了碘催化下双吲哚甲烷的 合成. 反应中催化量的碘作为 Lewis 酸活化苯甲醛的羰 基，使得具有亲核性的吲哚对羰基进行亲核加成，再经 过脱水、活化亚胺、Michael 加成等过程生成最终产物 (Scheme 17). 尽管其他含有金属离子的 Lewis 酸 $\left(\mathrm{InCl}_{3}\right.$, $\mathrm{LiClO}_{4}$ 等)也能完成该反应, 然而相比之下, 单质碘催化 的该反应收率更高，反应时间更短. 


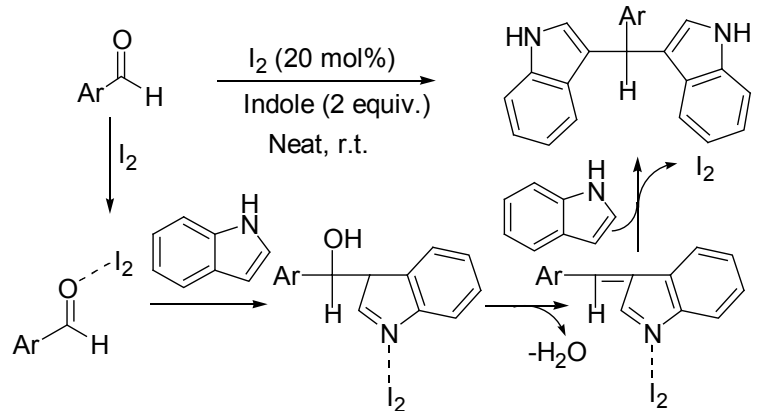

Scheme 17

\section{3 亲电性}

文献[3]已经提到, I- $\mathrm{I}$ 的键长为 $270 \mathrm{pm}$, 是目前已 知的最长的单键之一, 这导致了 $\mathrm{I}-\mathrm{I}$ 键能较弱 (150.7 $\mathrm{kJ} / \mathrm{mol}$ ), 当碘在靠近含有 $\pi$ 键的分子时, 两者由于电子 云的排斥作用会使碘分子极易发生极化, 从而导致 I- I 键的断裂 ${ }^{[44]}$. 此时, 如果分子中没有亲核试剂存在, 双 分子的碘即会加成到不饱和键中; 如果分子中含有其他 亲核性的官能团, 则会发生碘环化反应, 可以用来合成 各种杂环化合物 ${ }^{[6 a]}$. 碘环化反应早在 20 世纪就曾有报 道 ${ }^{[45]}$, 然而目前对于碘环化的详细机理却并未研究清 楚, 但通常人们认为经由以下两种途径发生: (1)经过一 个电荷转移复合物, 由于碘原子较大的极化率会与 $\pi$ 电 子相互诱导, 一方面促使 I一I 键断裂, 另一方面促使被 极化的不饱和键与亲核试剂反应, 生成环化产品; (2)不 饱和键的 $\pi$ 电子先与单质碘形成碘鎓离子中间体(碘鎓 离子是指含有碘正离子三元环的有机化合物, 尽管在 1937 年就曾推测该结构的存在, 但直到 1994 年才由 Brown 等 ${ }^{[46]}$ 首次用单晶证实, 他们在碘鎓离子三元环两 侧使用金刚烷骨架增大位阻降低了该中间体的活性从 而将其分离得到，由于其极高的活性特别容易与亲核试 剂反应得到环化产品(Scheme 18). 一般来讲, 分子内同 时含有不饱和键 (如烯烃, 联烯以及炔烃)和亲核试剂的 化合物，在一定条件下均可发生这类碘环化反应.

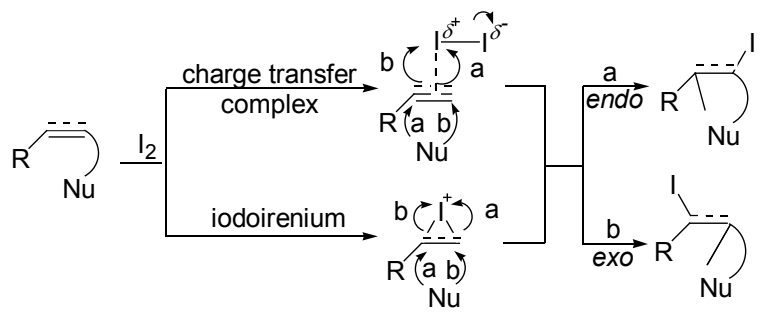

Scheme 18

对于烯烃的碘环化, 研究最早的是内酯化反应. 反 应通常是单质碘首先与双键发生作用, 羧基从背面进攻 活化的双键形成内酯环. 对于 $\gamma, \delta$-烯酸的碘环化反应， 许多研究者已对其关环的区域选择性和立体选择性做
了详细的研究 ${ }^{[47]}$. 一般来说, $\gamma, \delta$-烯酸主要通过 5-exo 关 环生成 $\gamma$ 内酯，而并非分通过 6-endo 关环的形式生成 $\delta$ 内酯(Scheme 19) ${ }^{[47 \mathrm{~d}]}$, 关环的选择性可以通过底物结构 进行调控 ${ }^{[47 f]}$, 也可以通过选择合适的催化剂来调控. 最 近 Burk 小组和 Jacobsen 小组 ${ }^{[48]}$ 分别使用手性 Lewis 碱 催化剂和叔胺脲催化剂完成了烯烃内酯化反应的对应 选择性合成，最高 $e e$ 值可达 $96 \%$. 除了羧酸之外，羧酸 盐和羧酸酯都能进行类似的反应. 另外，对于分子内存 在酰胺、硫酰胺、磺酰胺、羟基、 $\beta$-酮酸酯等亲核基团 的不饱和化合物, 在适当条件下均可发生分子内的关环 反应，生成相应的内酯、环醚，以及内酰胺 ${ }^{[49]}$.

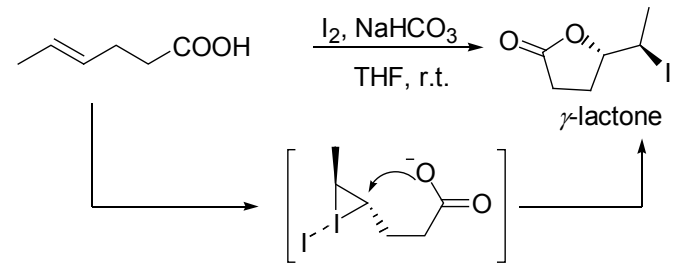

\section{Scheme 19}

联烯具有累积的 $C=C$ 双键结构, 并且相邻的 $C=C$ 双键轨道相互垂直. 尽管与孤立的碳碳双键稍有区别, 但也很容易发生亲电环化反应. 2005 年麻生明小组 ${ }^{[50]}$ 报 道了 2,3-联烯酸乙酯的碘环化反应专一性地生成五元 4碘呋喃酮(Eq. 14). 单质碘通过活化联烯的双键使羰基 氧原子能进行区域选择性的加成反应.

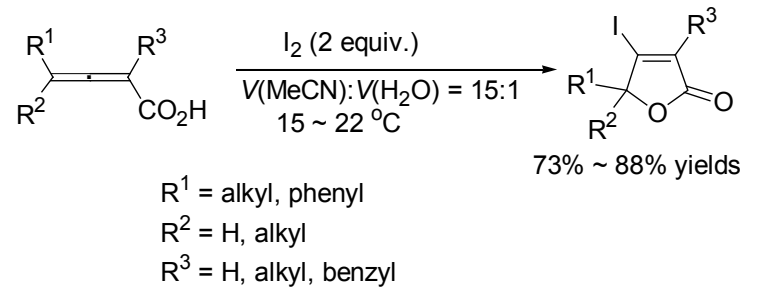

炔烃的碘环化反应是不饱和烃类碘环化反应中最 为重要的一类. 由于炔基在亲电环化后还保留一对双 键, 并且 Sonogshira 偶联为炔烃引入提供了极大的方便, 这成为合成杂芳环的先天条件. 当分子内含有氮亲核试 剂时, 可以得到吲哚、喹啉、异噁唑等含氮杂环; 当分 子中含有氧亲核试剂时，可以得到呋喃、吡喃、异香豆 素等含氧杂环; 当分子中含有硫亲核试剂时，可以得到 噻吩或苯并噻吩骨架. 关环方式在绝大多数情况下遵循 Baldwin 规则 ${ }^{[51]}$.

对于含氧杂环的构建，酚羟基是最常用作碘环化反 应的含氧亲核试剂. 1999 年 Arcadi 小组 ${ }^{[52]}$ 报道了以邻苯 乙炔基苯酚为底物，合成 3-磑代的苯并呋喃的结构 (Eq. 15). 然而如果底物炔基末端没有芳环，该反应仅仅得 
到苯酚被碘化的产品.

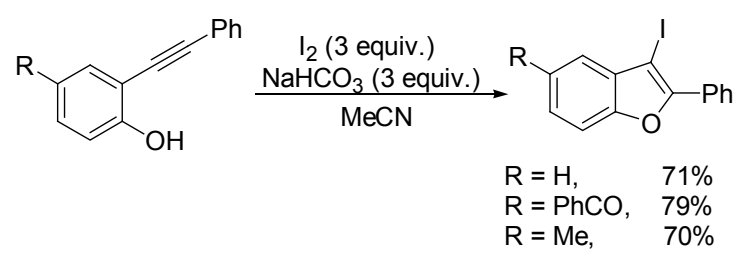

2002 年 Arcadi 小组 ${ }^{[53]}$ 为了构建呋喃吡啶的结构, 使用了邻位炔基的吡定酚醚完成了该转化(Eq. 16). 如 果酚羟基不被保护，则在底物在该条件下很容易分解变 质.<smiles>CCOc1cccnc1C#Cc1ccccc1</smiles>
$\frac{\mathrm{I}_{2} \text { (3 equiv.) }}{\underset{\mathrm{NaHCO}}{\mathrm{MeOH}_{3}(3 \text { equiv. })}}$<smiles>Ic1c(-c2ccccc2)oc2cccnc12</smiles>
$\begin{array}{ll}\mathrm{E}=\mathrm{CH}_{3} \mathrm{CO} & 76 \% \\ \mathrm{E}=\mathrm{PhCH}_{2} & 72 \%\end{array}$

3-炔基-1,2-二醇在碘和 $\mathrm{NaHCO}_{3}$ 的 $\mathrm{CH}_{2} \mathrm{Cl}_{2}$ 溶液中可 以以 5-endo-dig 的形式关环, 同时失去一分子水, 以较 高的收率得到 3-碘代呋喃 ${ }^{[54]}$. 由于呋喃结构 2 位的活性 较高, 使用通常碘化的方法只能在 2 位引入碘原子, 因 此该方法提供了一种合成 3-位碘代呋喃很好的策略 ${ }^{[55]}$. 当使用无取代的末端炔烃时不能得到环化的产品, 而是 得到碘对炔基加成的产物烯基二醇(Scheme 20).

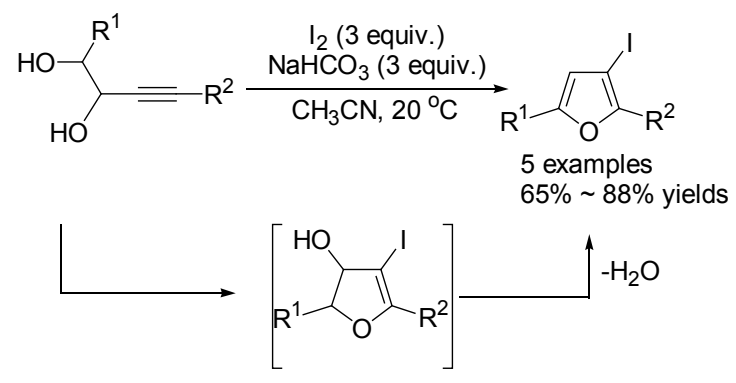

\section{Scheme 20}

烯酮也可以在外加亲核试剂的存在下发生碘环化 反应. 刘元红小组 ${ }^{[56]}$ 使用炔基烯酮作为反应底物, 以 $\mathrm{K}_{3} \mathrm{PO}_{4}$ 为碱, 通过加入亲核试剂(如苯酚、甲醇等), 在 Michael 加成的同时环化成 3-碘代呋喃(Scheme 21). 单 质碘通过活化炔烃诱导羰基的电子向参键转移, 从而增 强了烯酮 $\gamma$ 位的电正性，与亲核试剂发生加成反应.

2005 年 Larock 小组 ${ }^{[57]}$ 报道了一个碘环化合成异噁 唑的方法. 该小组将炔基酩做成肜醚，利用肟醚氧原子 的亲核性可以巧妙的合成 4-碘代异啞唑的骨架(Scheme 22). 该反应条件温和, 室温下即可进行, 产物中保留的 碘原子为以后通过偶联反应引入其他官能团提供了便 利.

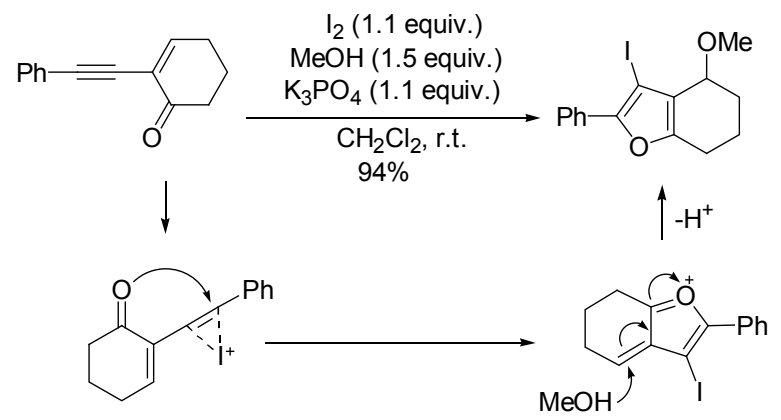

Scheme 21

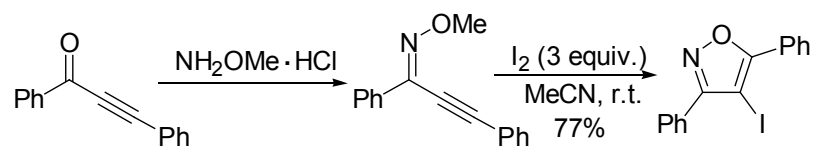

Scheme 22

除了羟基和羰基之外, 羧基、酯基也可作为亲核试 剂在碘环化反应中使用, 进而得到呋喃酮、吡喃酮以及 异香豆素类骨架结构. 此外, 2004 年丁贻祥小组 ${ }^{[58]}$ 报道 了一例使用磷酸酯作为亲核试剂, 利用炔基的碘环化反 应合成了磷原子取代碳原子的香豆素结构，这类化合物 具有较好的生物活性(Scheme 23). 当使用氯仿或二氯 甲烷为溶剂时, 6-endo-dig 关环顺利进行，产品的收率可 以达到 $80 \%$ 以上; 而当使用乙腈 $(\mathrm{MeCN})$ 或者 $N, N$-二甲 基甲酰胺(DMF)作溶剂时，只能分离得到参键加成一分 子碘的产物.
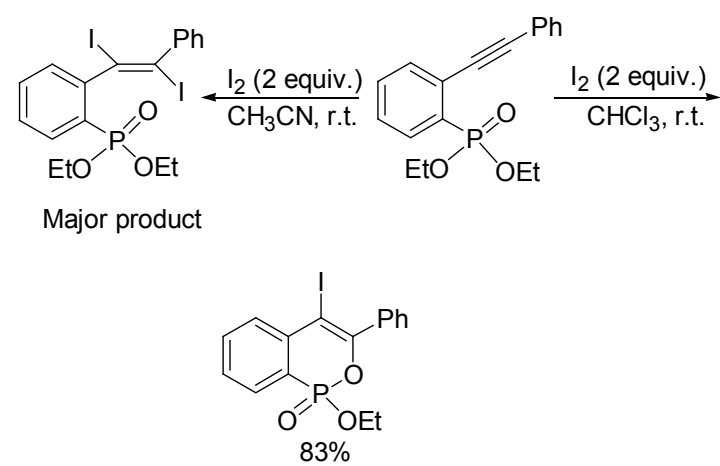

\section{Scheme 23}

含氮杂环的构建是碘环化反应中较为丰富的一类. 一般来讲, 脂肪胺的亲核性比相应脂肪醇的亲核性强. 从这个角度来说，苯胺要比苯酚更容易发生碘环化反 应. 然而事实上到目前为止，还没有苯胺或者脂肪胺发 生分子内碘环化反应的报道，其主要原因由于苯胺活性 较高，容易发生碘化反应或者氧化反应，但若将炔基苯 胺的氨基用酰基保护，降低了苯环的活性之后，则可以 发生碘环化反应生成相应的吲哚产品(Eq. 17) ${ }^{[59]}$. 
<smiles>[R]C#Cc1ccccc1N[AsH3]</smiles>
$\frac{\mathrm{I}_{2}(3 \text { equiv. })}{\mathrm{K}_{2} \mathrm{CO}_{3}(3 \text { equiv. })}$<smiles>[R]c1c(I)c2ccccc2n1S</smiles>

$\mathrm{R}=\mathrm{Ph}, \quad 95 \%$ $\mathrm{R}=\mathrm{Bu}, \quad 82 \%$

$\mathrm{R}=\mathrm{TMS}, 81 \%$

2004 年 Larock 小组 ${ }^{[60]}$ 报道了使用苯甲酰胺的炔基 衍生物为底物合成异吲哚啉酥. 实验证明, 该类底物在 进行碘环化的过程中反应选择性较好, 主要生成 5-exo 关环的结构(Eq. 18).<smiles>O=C(Nc1ccccc1)c1ccccc1C#Cc1ccccc1</smiles>

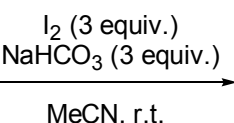<smiles>O=c1[nH]c(-c2ccccc2)c(I)c2ccccc12</smiles>

$10 \%$

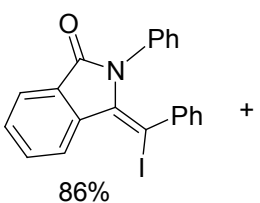

(18)
另外, 除了能够合成吲哚、异吲哚啉酮的结构, Knight 小组 ${ }^{[61]}$ 在 2007 年还报道了利用羟胺类底物构建 异啞唑啉. 反应使用 Ts 保护的羟胺, 同样使用 $\mathrm{I}_{2}$ 和 $\mathrm{K}_{2} \mathrm{CO}_{3}$ 在乙腈溶液中反应, 可以以 $89 \%$ 的收率得到 4 位 碘原子取代的异噁唑啉(Eq. 19).<smiles>[B]C#CC(ON[TeH])C(C)C</smiles>

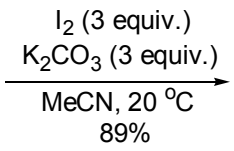<smiles>[3H]N1OC(C(C)C)C(I)=C1Br</smiles>

Flynn 小组 ${ }^{[62]}$ 使用邻甲氨基炔醇作为反应底物, 通 过对反应溶剂的调控可得到不同的杂环化合物(Scheme 24). 当底物在非质子性溶剂如乙腈或二氯中反应时, 专一性地得到 6-endo 关环的产品喹啉铵盐; 当底物在质 子性溶剂如乙醇或者甲醇中反应时, 专一性得到 5-exo 关环的产品 2-酰基吲哚.
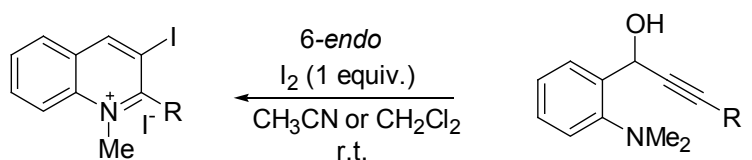

$71 \% \sim 89 \%$ yields
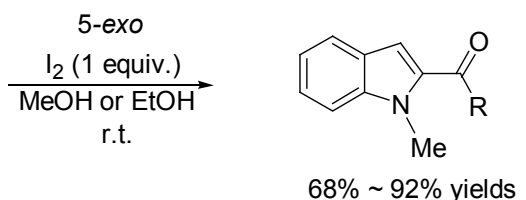

Scheme 24

在 Flynn 小组的体系中, 尽管能高效地得到喹啉骨
架的结构, 但是这种结构只能以喹啉盐的形式分离出 来，为了将这种合成方式变得更加实用，梁永民小组结 合以上几种含氮亲核试剂以及选择性的优势，在 2011 年发表了另外一篇代表性的工作 ${ }^{[63]}$, 反应选用对甲苯 磺酰胺(NHTs)为亲核试剂，受体选用炔丙基叔醇，以甲 醇为溶剂, 可以较高收率得到 3-碘代的喹啉衍生物(Eq. 20), 避免了使用 $N, N$-二甲基苯胺产生难以分离的喹啉 盐产品. 尽管所使用的溶剂是质子性溶剂，但是没有观 察到 5-exo-dig 关环的产品，因此亲核试剂的结构也是 影响关环方式的重要因素.

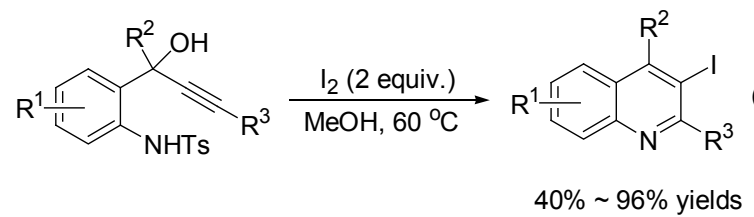

除此之外, Larock 小组 ${ }^{[64]}$ 还使用亚胺作为亲核试剂 进行环化反应，所用的亚胺是叔丁基胺与苯甲醛脱水生 成的，由于关环后形成的异喹啉铵盐不稳定，在碱的存 在下叔丁基以异丁烯的形式离去从而生成相应的异喹 啉环(Scheme 25). 只有使用 $\mathrm{NaOCO}_{2} \mathrm{CH}_{3}$ 为碱来促进该 反应进行时, 可以专一性得到异喹啉结构, 而换成其他 有机或无机碱则会产生副反应或者破坏底物的亚胺结 构.

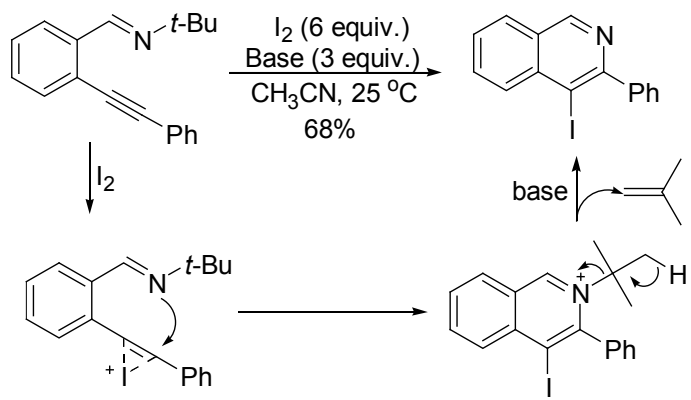

\section{Scheme 25}

2008 年 Yamamoto 小组 ${ }^{[65 a]}$ 使用叠氮作为亲核试剂, 室温下进行炔烃的碘环化反应，可以得到 4-碘代的异喹 啉结构(Scheme 26). 当炔烃末端变成缺电子的芳环体 系(如吡啶), 反应只能得到双碘化的烯烃. 2010 年该小 组 ${ }^{[65 b]}$ 又将该方法应用于 4 -碘代喹啉的合成上，也得到 了不错的效果. 对于此两类杂环, 该小组 ${ }^{[66]}$ 曾在 2009 年使用 $\mathrm{AuCl}_{3} / \mathrm{AgSbF}_{6}$ 催化体系进行合成研究，尽管可 以得到未被碘化的异喹啉及喹啉结构, 但是收率仅为中 等 $(60 \% \sim 80 \%)$, 且所需反应温度较高 $\left(100{ }^{\circ} \mathrm{C}\right)$. 因此, 通过与过渡金属催化剂的反应对比，体现了单质碘在炔 烃亲电环化反应中的优势. 


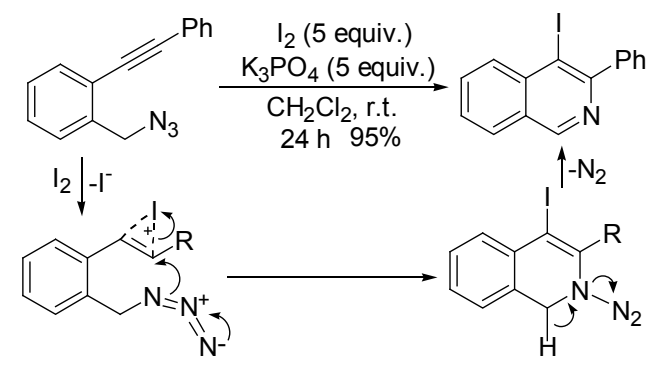

Scheme 26

同样，使用含硫亲核试剂也可以在单质碘的作用下 构建环丁砜、环丁硫醚、噻吩及苯并噻吩等含硫的杂环 结构 ${ }^{[67]}$. Larock 小组 ${ }^{[67 a]}$ 利用含有邻位炔基的苯硫醚, 合 成了 3-碘代的苯并噻吩骨架化合物(Eq. 21).
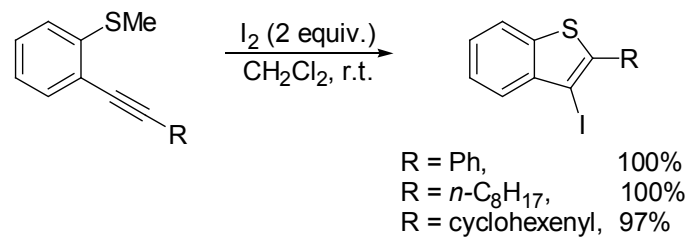

分子内如果含有较强的碳亲核试剂, 也可以用碘环 化策略合成比较重要的碳环骨架. 早在 1993 年, Taguchi 小组 ${ }^{[68]}$ 报道了一例碘环化合成五元碳环的方法. 该方 法使用 $\mathrm{Ti}(\mathrm{O} t-\mathrm{Bu})_{4}$ 活化羰基化合物, 提高其亲核性, 进 而与单质碘活化后的炔烃发生分子内的环化反应. 2009 年 Wirth 小组 ${ }^{[69]}$ 将该底物拓展到具有苯环结构的碳链 中, 使用了邻位炔基的芳基丙二酸酯为底物, 通过碘环 化反应得到了 3-碘代的狮(Eq. 22).

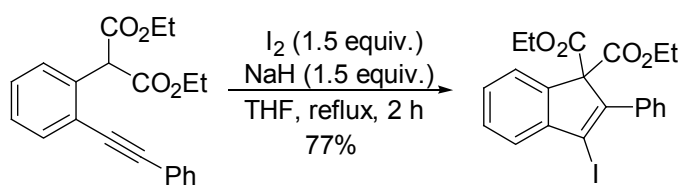

一般来讲, 芳环上不含强的供电子基时亲核性较 弱, 但仍然可以与活化的炔烃进行反应. 通过单质碘活 化参键而提高其亲电性, 可以顺利发生苯环与炔烃的 $\mathrm{C}-\mathrm{C}$ 成键反应. 2005 年, Larock 等 ${ }^{[70]}$ 利用单质碘活化炔 酮成功地在苯环的一侧构筑了碘代荎酚结构(Eq. 23).<smiles>O=C(C#Cc1ccccc1)Cc1ccccc1</smiles>

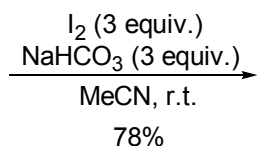<smiles>Oc1cc2ccccc2c(-c2ccccc2)c1I</smiles>

烯胺 $\alpha$ 碳原子亲核活性较高, 可以与炔基进行碘环 化的反应. 2010 年姚祝军小组 ${ }^{[71]}$ 报道了利用炔丙基烯胺 的底物分子内碘环化反应合成吡啶并萗醌 (Azaanthraquinone)的结构, 该结构是许多生物碱中的基
本骨架.

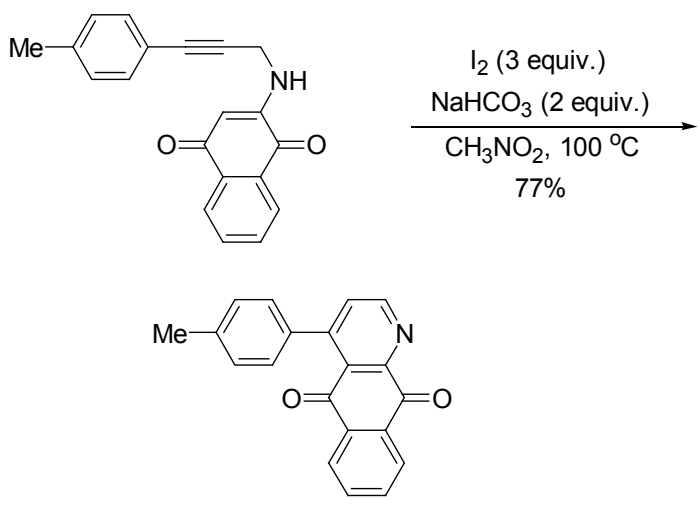

吲哚 2 位也是亲核活性较高的位点. 2011 年 Rustagi 等 ${ }^{[72]}$ 巧妙地利用该位点与炔烃进行碘环化反应, 得到 了吲哚并喹啉的结构(Scheme 26). 这种四环含氮稠环 结构被认为是优良的刚性分子平台, 在主客体化学、液 晶化学等领域具有重要应用.

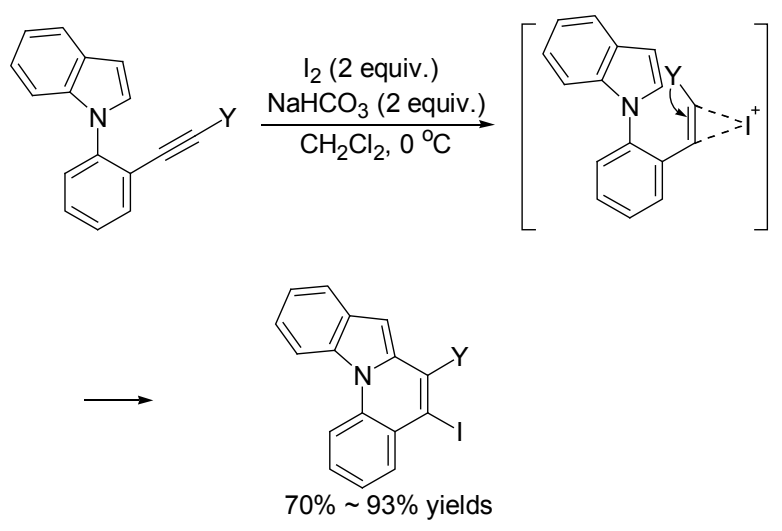

Scheme 27

以上的例子证明，含氮、氧、硫、碳亲核试剂在合 适的条件下都能发生碘环化反应，得到相应的芳香骨架 结构. 为了更深入的了解亲电碘环化反应的特点, 2009 年 Larock 小组 ${ }^{[73]}$ 详细总结了几类亲核试剂发生碘环化 反应的活性顺序(Scheme 28), 一般来讲, 含硫亲核试剂
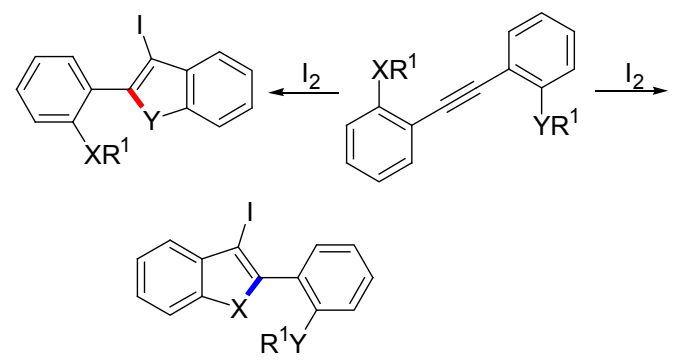

$\mathrm{SMe}>\mathrm{CO}_{2} \mathrm{Me}>\mathrm{NMe}_{2}>\mathrm{Aryl}>\mathrm{OMe}>\mathrm{OBn} ; \mathrm{CH}=\mathrm{Nt}-\mathrm{Bu}>\mathrm{OMe}>$ $\mathrm{OAc}$; $\mathrm{CONHPh}>\mathrm{CO}_{2} \mathrm{Me}<\mathrm{CHO}$

Scheme 28 
的活性最高, 其次是含氮亲核试剂, 含氧亲核试剂活性 最弱, 而含碳亲核试剂的活性则主要跟其结构有关.

另外, 近几年来碘环化反应有了新的发展一一碘环 化形成的杂环中间体具有较高反应活性的位点, 在适当 的条件下可以接着发生后续的各种化学反应, 这样就可 以一次构筑两个以上的芳环, 具有极高的合成效率, 这 种策略通常被称为碘环化诱导的串联环化反应 ${ }^{[74]}$. 例 如, 两次碘环化的串联反应, 首先通过碘环化反应生成 一个杂环系(如吲哚、噻吩), 所生成的杂环具有一个较 强的碳原子亲核位点, 分子内若存在还可以继续被单质 碘活化的不饱和键, 并且恰好可以与杂环的活性位点之 间形成热力学稳定的五元或者六元环系, 那么可以发生 第二次碘环化. 例如汪清民小组 ${ }^{[74 a]}$ 构建吲哚并哌啶环 系的策略、吴明忠小组 ${ }^{[74 b]}$ 构建苯并咔唑的策略(Scheme 29) 以及 Yamamoto 小组 ${ }^{[74 c]}$ 报道的苯并噻吩并萗的策 略都是二次串联碘环化反应的典型代表. 此外, 碘环化 之后生成的杂环中间体也可以继续发生 $[3+2]$ 环加成反 应，生成结构复杂的桥环骨架，梁永民小组 ${ }^{[74 \mathrm{~d}]}$ 报道的 氧杂 $[3,2,1]$ 环系就是该方法的典型代表(Scheme 30).
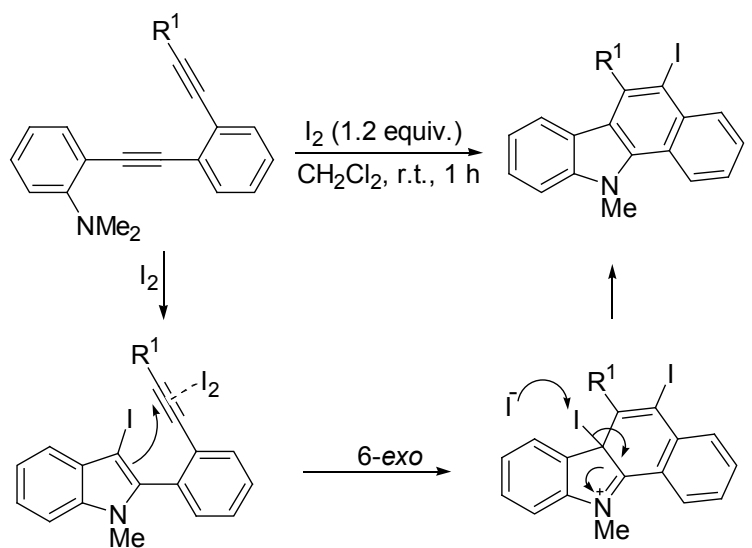

Scheme 29

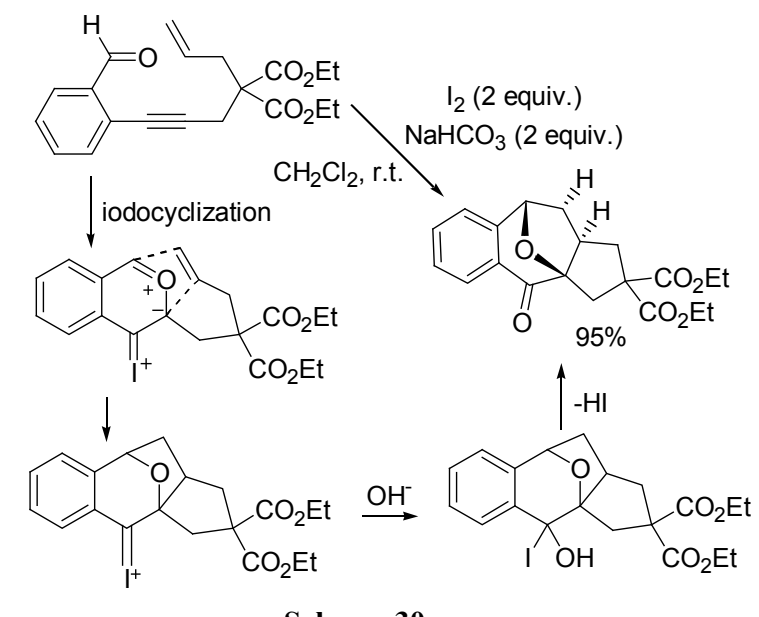

Scheme 30

在以上这些构筑杂环化合物的碘环化反应中, 有些
反应只在碘单质的存在下就可以进行 ${ }^{[50,57,58,62,63]}$, 还有 一些反应则需要碱的参与 $\left(\mathrm{Na}_{2} \mathrm{CO}_{3}, \mathrm{NaHCO}_{3}, \mathrm{~K}_{3} \mathrm{PO}_{4}\right.$ 等). 碱的加入可以促使单质碘形成亲电性更强的 “ $\mathrm{I}^{+}$” 中间 体，有利于碘环化反应的发生.

\section{4 多功能活化}

上文总结了单质碘在有机合成中常用的 3 种反应性 质，并对每一类性质的代表性反应进行了介绍. 然而在 某些转化中，单质碘的 3 种性质可以相互配合，共同活 化底物从而完成复杂转化.

2012 年 Batra 小组 ${ }^{[75]}$ 报道了一例利用 $\mathrm{I}_{2} / \mathrm{K}_{2} \mathrm{CO}_{3}$ 体系 合成取代喹啉的方法(Scheme 31). 在该反应中单质碘 起 3 个作用: 首先作为 Lewis 酸活化羰基完成氨基的 Michael 加成反应形成 $\mathrm{C}-\mathrm{N}$ 键; 接着在 $\mathrm{I}_{2}$ 和 $\mathrm{K}_{2} \mathrm{CO}_{3}$ 的 共同作用下在羰基 $\alpha$ 位发生碘化, 并进一步脱除 $\mathrm{HI}$ 重 新形成芳环; 最后, 单质碘发挥氧化脱氢作用将二氢喹 啉氧化成喹啉. 其中, 后两个作用需要消耗两分子的单 质碘，而活化羰基并不需要单质碘的消耗.

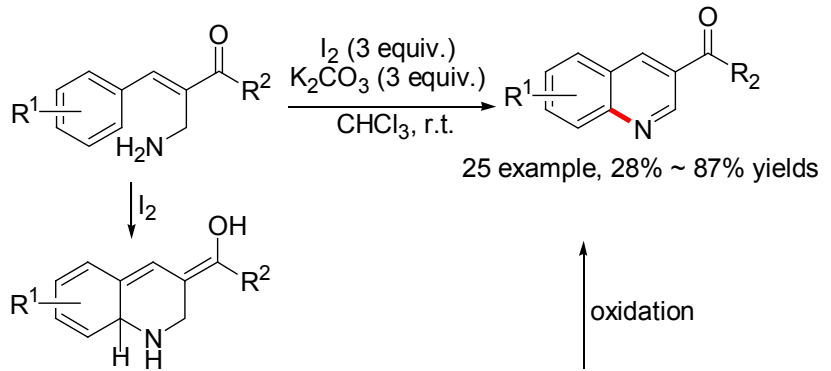<smiles>[R]C(=O)C1=Cc2cc[Y10]([H])cc2NC1</smiles>

Scheme 31

Punniyamurthy 等 ${ }^{[76]}$ 使用伯胺和 1,3-烯炔为底物, 在单质碘的作用下合成五取代的吡咯结构(Scheme 32).

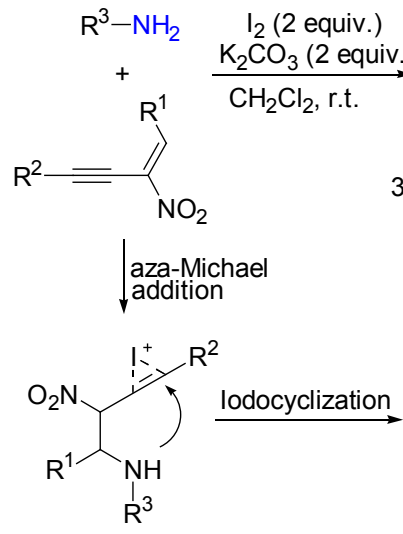

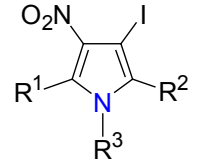

33 examples, up to $83 \%$ yield

Oxidative aromatization<smiles>[R]C1=C(I)C([N+](=O)[O-])C([R])N1[R]</smiles>

Scheme 32 
在该反应中单质碘也发挥了 3 个作用: 一是 Lewis 酸活 化硝基促进胺对硝基烯烃的氮杂 Michael 加成反应; 二 是亲电活化炔烃完成胺与炔烃之间的碘环化反应; 三是 氧化脱氢将二氢吡咯转化成吡咯结构. 该反应条件温 和, 底物范围较广。

我们小组 ${ }^{[77]}$ 最近报道的以芐胺和 $\alpha$-溴代酮为底物, 在单质碘的作用下可以合成多取代的噁唑(Scheme 33). 芐胺与溴代酮反应生成的 $\alpha$-氨基酮, 首先被单质碘氧化 形成亚胺, 接着单质碘催化烯醇负离子对亚胺的分子内 亲核加成, 最后将噁唑啉氧化脱氢形成噁唑. 该方法高 效实用, 可以一步完成天然产物 Halfordinol 的全合成. 在该反应中单质碘发挥了氧化脱氢和 Lewis 酸的双重作 用.

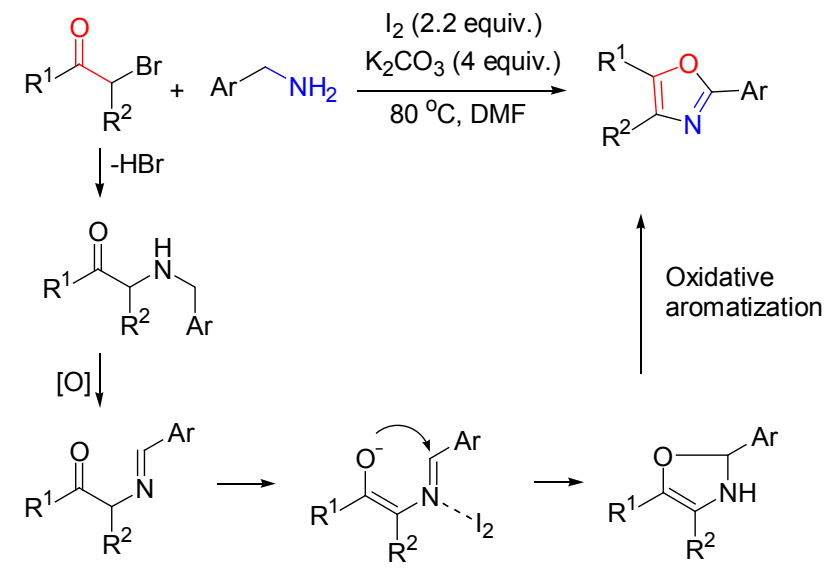

Scheme 33

吴安心小组 ${ }^{[78]}$ 最近报道了使用芳基甲基酮和芐胺 为底物, 以 DMSO 为溶剂, 也可以通过单质碘的多功能 活化一步得到 2,5-二取代噁坐(Scheme 34). 该反应不需 要预先制备 $\alpha$-卤代酮，因而大大缩短了合成噁唑的步 骤. 在单质碘的作用下芳基甲基酮可以原位生成 $\alpha$-碘代 酮, 并进一步被单质碘氧化为 $\alpha$-羰基醛, 通过与苄胺的

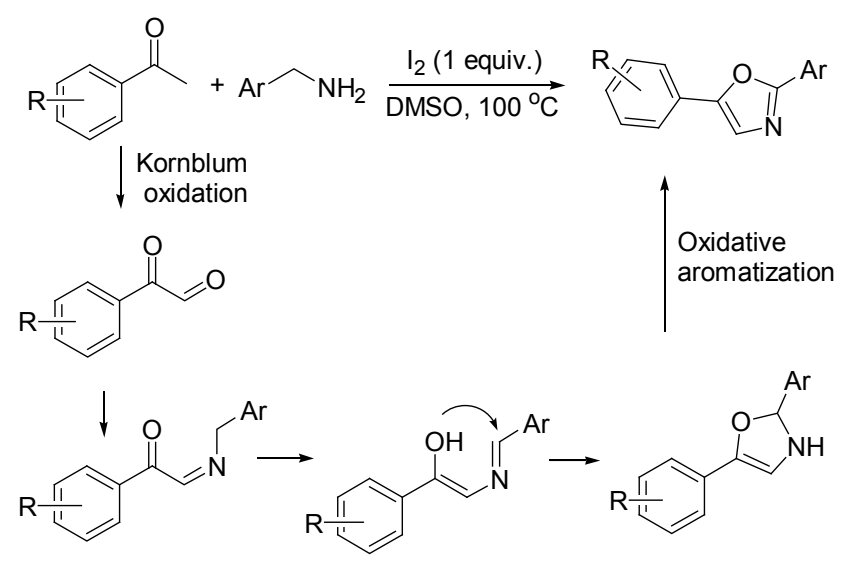

Scheme 34
缩合, 分子内环合以及噁唑啉的氧化等过程可高收率地 得到相应的 2,5-二取代噁唑.

\section{5 作为氧化反应的催化剂}

一般来说，单质碘属于弱氧化剂，有些反应单独使 用单质碘无法完成. 近些年来, 许多课题组通过使用其 他活性更高的氧化剂配合催化量的单质碘，完成了一些 丰富多彩的转化.

首先值得一提的是 $\mathrm{O}_{2} / \mathrm{I}_{2}$ 体系. 氧气作为一种理想 的绿色氧化剂, 配合催化量的单质碘, 既能完成氧化成 键又能继续氧化脱氢形成芳香环. 江焕峰小组 ${ }^{[79]}$ 使用 简单酮为底物和芐胺或者硫酰胺, 完成了咪唑和噻唑等 杂环的合成(Scheme 35); Prabhu 小组 ${ }^{[80]}$ 则使用该体系完 成了四氢异喹啉芐位 $\mathrm{C}-\mathrm{H}$ 键的活化反应.
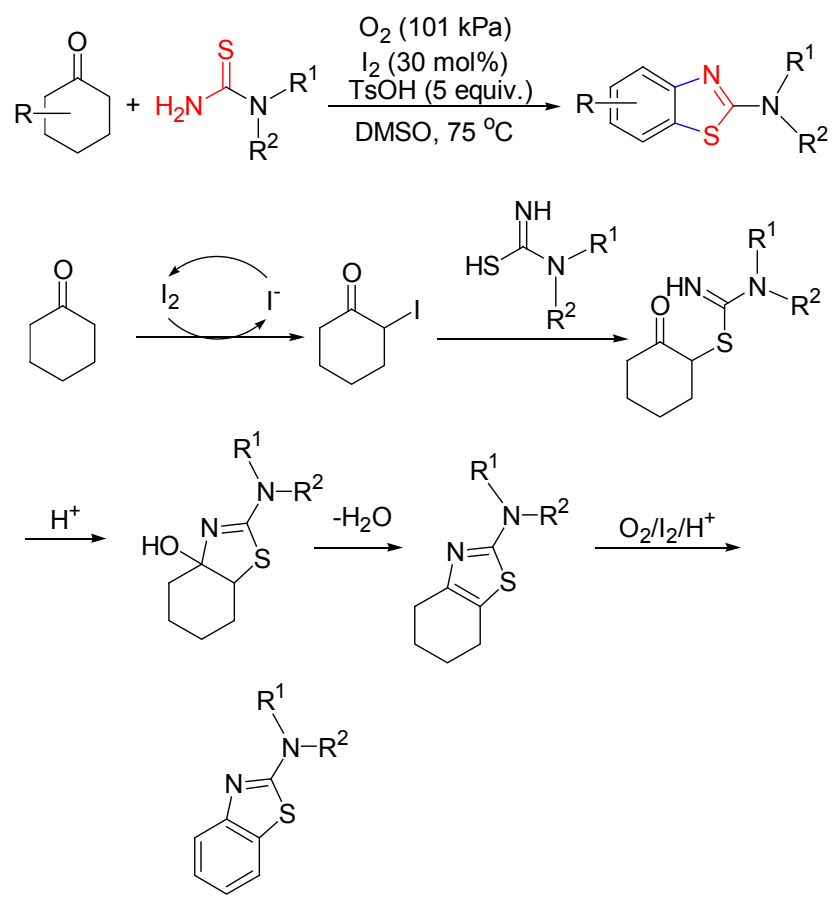

Scheme 35

过氧叔丁醇(TBHP)也是一类绿色氧化剂. 汪志勇 小组利用 $\mathrm{TBHP} / \mathrm{I}_{2}$ 组合发展了多种杂环化合物包括噁 唑 ${ }^{[81]}$ 、喹啉 ${ }^{[82]}$ 、吡啶 ${ }^{[83]}$ 、喹唑啉 ${ }^{[84]}$ 在内的含氮骨架的 构筑方法. 此外, 许多官能团的转化及 $\mathrm{C}-\mathrm{H}$ 活化等反 应也可以利用 $\mathrm{TBHP} / \mathrm{I}_{2}$ 体系完成 ${ }^{[85,86]}$. 例如, 在碱的存 在下使用甲酰胺为胺源, $\mathrm{TBHP} / \mathrm{I}_{2}$ 可以将芐醇或者烯丙 基醇转化成相应的酰胺(Eq. 25$)^{[85]}$.

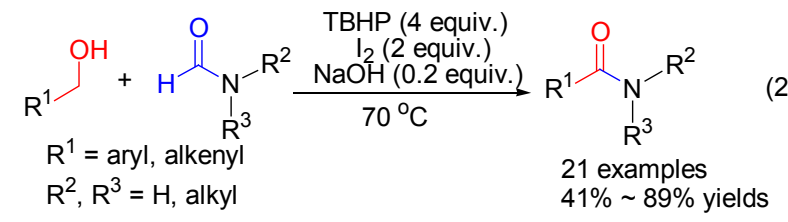


双氧水 $\left(\mathrm{H}_{2} \mathrm{O}_{2}\right)$ 与单质碘一样, 也属于较为温和的氧 化剂. 相对于 TBHP 而言, $\mathrm{H}_{2} \mathrm{O}_{2} / \mathrm{I}_{2}$ 组合促进的氧化反应 报道并不多. 2013 年 Itoh 小组 ${ }^{[87]}$ 使用该体系完成了叔胺 $\alpha$-位与碳亲核试剂之间的 $\mathrm{C}-\mathrm{C}$ 成键反应，这是首例报 道的单质碘催化 $\mathrm{sp}^{3}-\mathrm{C}-\mathrm{H}$ 键之间脱氢偶联反应(Eq. 26).<smiles>N#[N+]N1CCc2ccccc2C1</smiles>

$\mathrm{Nu}-\mathrm{H}: \mathrm{MeNO}_{2}, \mathrm{EtNO}_{2}$, $\mathrm{CH}_{2}\left(\mathrm{CO}_{2} \mathrm{Me}\right)_{2}$, $\mathrm{MeCOMe}, i$-BuCOMe<smiles>C1CCCOCC1</smiles><smiles>[Y]N1CCc2ccccc2C1[N]</smiles>

18 examples up to $93 \%$ yield
2014 年, 吴安心小组 ${ }^{[88]}$ 使用芳香甲基酮和苯乙胺 为底物, 在 $\mathrm{H}_{2} \mathrm{O}_{2} / \mathrm{I}_{2}$ 共同作用下可以一步得到异喹啉的 结构. 如果将苯乙胺换成色胺, 可以得到 $\alpha$-咔啉结构的 化合物, 该方法为含有咔啉结构的天然产物 (如 Fascaplysin 及 Papaverin)的合成提供了便利. 反应首先 利用 Kornblum 氧化将芳香甲基酩氧化形成 $\alpha$-羰基醛, 接着醛基与伯胺缩合, 并继续与芳环发生傅克反应形成 六元含氮杂环, 在单质碘的进一步氧化下脱氢形成最终 的芳香结构(Eq. 27).

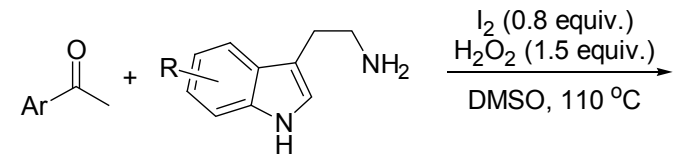<smiles>O=C(c1ccccc1)c1nccc2c1[nH]c1ccccc12</smiles>

33 examples up to $95 \%$ yield

$N$-溴代丁二酰亚胺(NBS) 是能产生活性溴正离子的 试剂, 通过产生的溴正离子来发挥氧化作用. 2011 年李 志平小组 ${ }^{[89}$ 报道了使用 $\mathrm{NBS} / \mathrm{I}_{2}$ 组合合成 2,3 -二取代吲 哚的反应(Eq. 28). 在该体系中, 使用催化量单质碘用来 产生自由基来促进 NBS 氧化的进行, 他们通过控制实 验表明，没有单质碘的加入反应不能发生.
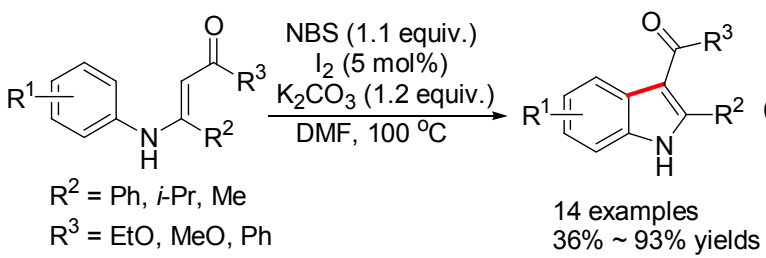

高价碘与单质碘的组合也是备受关注的一类反应， 其中最值得一提的是二醋酸碘苯 $(\mathrm{PIDA}) / \mathrm{I}_{2}$ 组合所诱导 的远程官能团化反应. PIDA 与 $\mathrm{I}_{2}$ 反应会生成碘自由基,
当分子内存在的含氮或者含氧的官能团时，碘自由基会 与氮原子或氧原子上的孤对电子反应生成含氮或含氧 自由基, 这类自由基会选择性地与分子内的活性位点氢 (叔碳、氧原子邻位或者氮原子邻位)反应形成 $\mathrm{C}-\mathrm{N}$ 键 或者 $\mathrm{C}-\mathrm{O}$ 键. 例如, Suárez 小组 ${ }^{[90]}$ 使用 $\mathrm{PIDA} / \mathrm{I}_{2}$ 的组合 区域选择性地在环醚的邻位形成 $\mathrm{C}-\mathrm{O}$ 键, 产物比例需 要由 R 取代基的选择进行控制(Eq. 29).
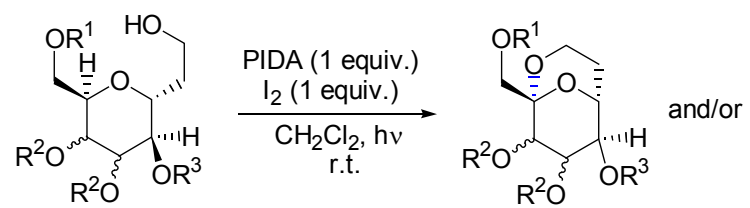

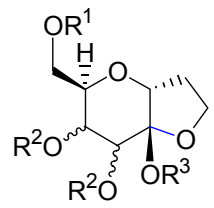

相比较而言, $\mathrm{PIDA} / \mathrm{I}_{2}$ 诱导形成 $\mathrm{C}-\mathrm{N}$ 的反应要更为 丰富，不管是氨基还是保护后的酰胺，都可以发生此类 反应 ${ }^{[91]}$. 2011 年 Lee 小组 ${ }^{[91 \mathrm{~d}]}$ 将该策略应用在 Cephalostatin 类似物的合成中，可以立体专一性地得到 目标产品(Eq. 30).
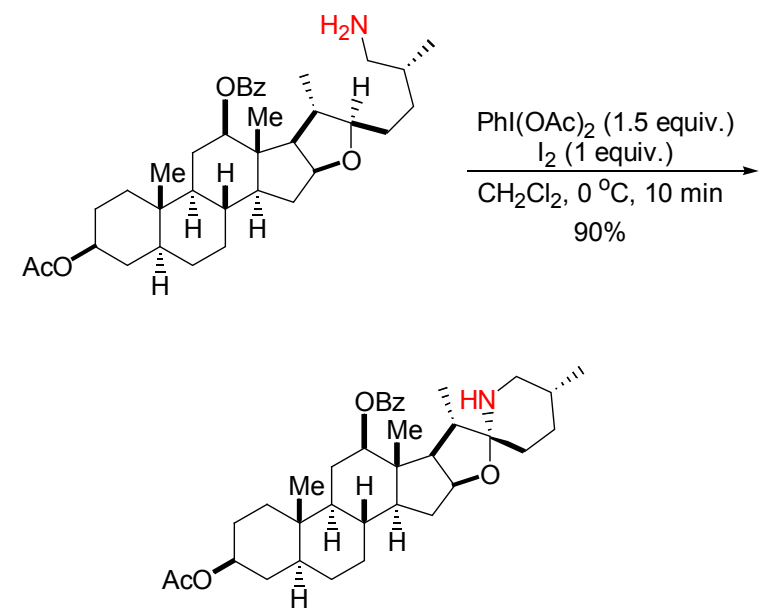

IBX 也是广泛使用的一类高价碘试剂, 它与单质碘 反应可以将烯烃转化为 $\alpha$-碘代酮 ${ }^{[92 a]}$. 2010年, 吴安心小 组 ${ }^{[92 b]}$ 利用该体系一锅法合成了 1,4 -烯二酮化合物 (Scheme 36).

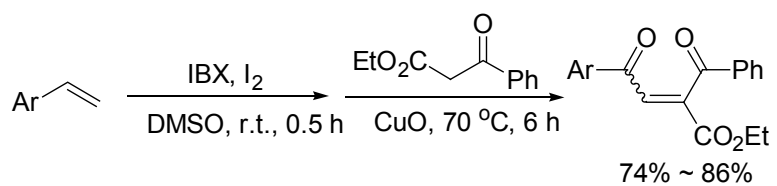

Scheme 36 
此外, 金属氧化剂也经常配合单质碘使用, 如 $\mathrm{CuO} / \mathrm{I}_{2}$ 的体系在有机合成中也是比较常见的组合. 该组 合的主要作用是以较高的收率快速构建 $\alpha$-碘代酮, 并通 过 DMSO 氧化成 $\alpha$-羰基醛, 与各种亲核试剂发生串联 多组分反应(Scheme 37) ${ }^{[93]}$.

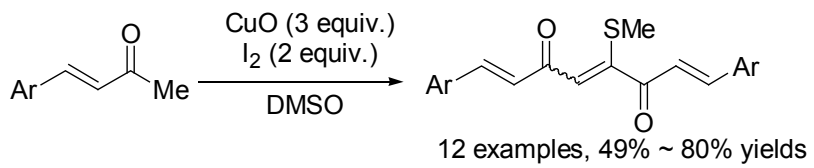

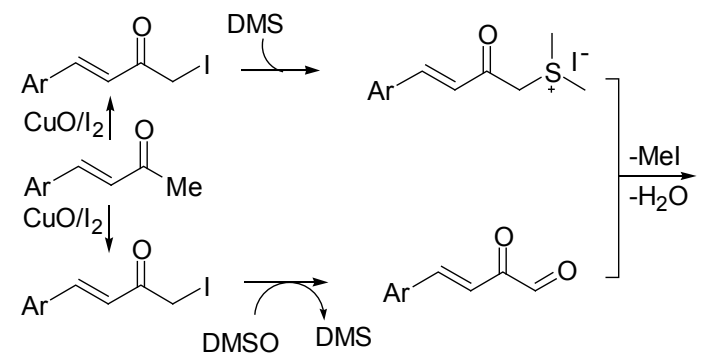

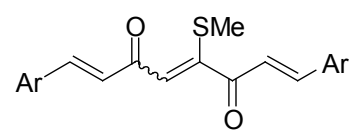

Scheme 37

Oxone 是商品化的过硫酸氢钾复合盐, 也是有机合 成中经常使用的一类氧化剂. 2013 年 Nachtsheim 小组 ${ }^{[94]}$ 使用 Oxone $/ \mathrm{I}_{2}$ 的组合可以完成甲基酮与氨基酸生成多取 代啞唑的反应(Scheme 38). 其中氨基酸的羧基可以在 该体系下脱除, 从而将氨基酸的氮原子引入到啞唑骨架 内.

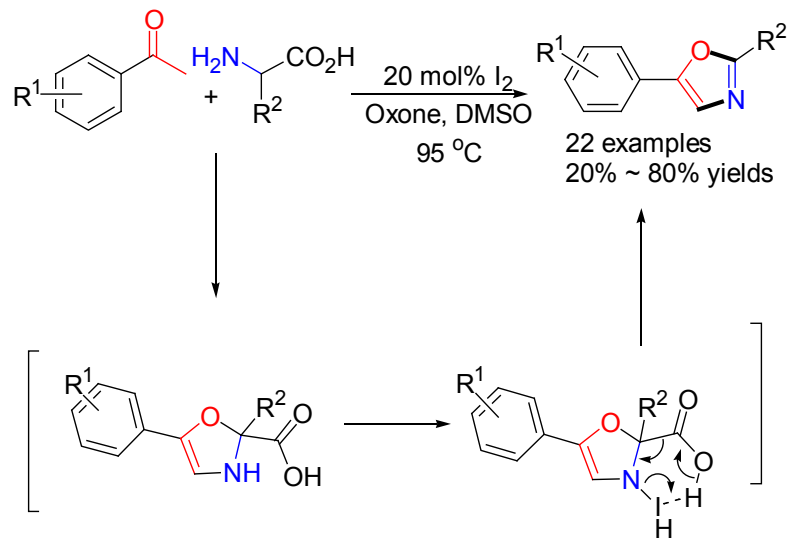

Scheme 38

\section{6 结论与展望}

本文对单质碘在有机合成中的三类基本反应性一
一氧化性、酸性和亲电性进行了系统的概括和总结，并 结合单质碘的结构及相关文献对其相应的化学性质进 行了解释, 同时对单质碘在复杂转化中综合发挥 3 种作 用进行多功能活化的报道进行了介绍. 此外, 由于单质 碘是一类简单廉价, 绿色高效的催化剂, 本文还总结了 常见氧化剂配合单质碘完成的多种化学转化, 重点对近 5 年来在杂环合成、偶联反应、 $\mathrm{C}-\mathrm{H}$ 官能团转化等方 向的研究进行了总结. 值得一提的是, 到目前为止, 单 质碘促进的某些转化的机理并未得到相应的实验证实, 许多新型反应也有待化学家们的进一步探索和开发, 未 来单质碘在有机合成领域中的应用仍然充满着机遇和 挑战.

致谢 感谢南开大学张弛教授对本文部分内容的指导 和建议.

\section{References}

[1] Swain, P. A. Bull. Hist. Chem. 2005, 30, 103.

[2] (a) Togo, H.; Iida, S. Synlett 2006, 2159.

(b) Finkbeiner, P.; Nachtsheim, B. J. Synthesis 2013, 45, 979.

[3] Leal, J. P.; Marques, N.; Takats, J. J. Organomet. Chem. 2001, 632, 209.

[4] Svensson, P. H.; Kloo, L. Chem. Rev. 2003, 103, 1649.

[5] (a) Stanbury J. B. In Endemic Goitre, World Health Organization, Geneva, 1960, pp. 261 262 .

(b) Vitamine and mineral requirements in human nutrition. In Iodine, 2nd ed, World Health Organization, Geneva, 2004, pp. 303 304.

[6] (a) Godoi, B.; Schumacher, R. F.; Zeni, G. Chem. Rev. 2011, 111, 2937.

(b) Parvatkar, P. T.; Parameswaran, P. S.; Tilve, S. G. Chem. Eur. J. 2012, 18, 5460 .

[7] (a) Zhou, Y.; Yan, P.-F.; Li, G.-M.; Chen, Z.-J. Chin. J. Org. Chem. 2009, 29,1719 (in Chinese).

(周颖，闰鹏飞，李光明，陈正军，有机化学, 2009, 29, 1719.)

(b) Wang, H.-S.; Miao, J.-Y.; Zhao, L.-F. Chin. J. Org. Chem. 2005, 25, 615 (in Chinese).

(王宏社, 苗建英, 赵立芳, 有机化学, 2005, 25, 615.)

[8] Jereb, M.; Vražič, D.; Zupan, M. Tetrahedron 2011, 67, 1355.

[9] Das, S.; Borah, R.; Devi, R. R.; Thakur, A. J. Synlett 2008, 2741.

[10] Ren, Y.-M.; Cai, C.; Yanga, R.-C. RSC Adv. 2013, 3, 7182.

[11] Mphahlele, M. J. Molecules 2009, 14, 5308.

[12] Küpper, F. C.; Feiters, M. C.; Olofsson, B.; Kaiho, T.; Yanagida, S.; Zimmermann, M. B.; Carpenter, L. J.; Luther III, G. W.; Lu, Z.; Jonsson, M.; Kloo, L. Angew. Chem., Int. Ed. 2011, 50, 11598.

[13] (a) Yamada, S.; Morizono, D.; Yamamoto, K. Tetrahedron Lett. 1992, 33, 4329.

(b) Yamamoto, K.; Shimizu, M.; Yamada, S. J. Org. Chem. 1992, 57,33 .

[14] Mori, N.; Togo, H. Synlett 2005, 1456.

[15] Ellman, G. L. Arch. Biochem. Biophys. 1959, 82, 70.

[16] Doi, J. T.; Musker, W. K. J. Am. Chem. Soc. 1981, 103, 1159.

[17] (a) Mallory, F. B.; Mallory, C. W. Org. React. 1984, 30, 1.

(b) Yamato, T.; Miyamoto, S.; Hironaka, T.; Miura, Y. Org. Lett. 2005, 7, 3 .

[18] Yeh, H.; Yeh, S.; Chen, C. Chem. Commun. 2003, 2632. 
[19] (a) Zuo, Z.; Xie, W.; Ma, D. J. Am. Chem. Soc. 2010, 132, 13226. (b) Zuo, Z.; Ma, D. Angew. Chem., Int. Ed. 2011, 50, 12008.(c) Zi, W.; Xie, W.; Ma, D. J. Am. Chem. Soc. 2012, 134, 9126.

(d) Teng, M.; Zi, W.; Ma, D. Angew. Chem., Int. Ed. 2014, 53, 1814.

[20] (a) Zhu, Y.; Liu, M.; Jia, F.; Yuan, J.; Gao, Q.; Lian, M.; Wu, A. Org. Lett. 2012, 14, 3392.

(b) Zhu, Y.; Jia, F.; Liu, M.; Wu, L.; Cai, Q.; Gao, Y.; Wu, A. Org. Lett. 2012, 14, 5378.

(c) Yang, Y.; Gao, M.; Zhang, D.-X.; Wu, L.-M.; Shu, W.-M.; Wu, A.-X. Tetrahedron 2012, 68, 7338.

[21] Gao, Q.; Wu, X.; Liu, S.; Wu, A. Org. Lett. 2014, 16, 1732.

[22] Yin, G.; Zhou, B.; Meng, X.; Wu, A.; Pan, Y. Org. Lett. 2006, 8, 2245.

[23] Zhu, Y.; Fei, Z.; Liu, M.; Jia, F.; Wu, A. Org. Lett. 2013, 15, 378.

[24] Fei, Z.; Zhu, Y.-P.; Liu, M.-C.; Jia, F.-C.; Wu, A.-X. Tetrahedron Lett. 2013, 54, 1222.

[25] Cao, L.; Ding, J.; Gao, M.; Wang, Z.; Li, J.; Wu, A. Org. Lett. 2009, 11, 3810 .

[26] (a) Li, Y.-X.; Ji, K.-G.; Wang, H.-X.; Ali, S.; Liang, Y.-M. J. Org. Chem. 2011, 76, 744.

(b) Li, Y.-X.; Wang, H.-X.; Ali, S.; Xia, X.-F.; Liang, Y.-M. Chem. Commun. 2012, 48, 2343.

[27] Wu, W.-B.; Huang, J.-M. Org. Lett. 2012, 14, 5832.

[28] Gao, W.-C.; Jiang, S.; Wang, R.-L.; Zhang, C. Chem. Commun. 2013, 49, 4890.

[29] Yu, W.; Huang, G.; Zhang, Y.; Liu, H.; Dong, L.; Yu, X.; Li, Y.; Chang, J. J. Org. Chem. 2013, 78, 10337.

[30] Sawangphon, T.; Katrun, P.; Chaisiwamongkhol, K.; Pohmakotr, M.; Reutrakul, V.; Jaipetch, T.; Soorukram, D.; Kuhakarn, C. Synth. Commun. 2013, 43, 1692.

[31] Gao, Q.; Wu, X.; Jia, F.; Liu, M.; Zhu, Y.; Cai, Q.; Wu, A. J. Org. Chem. 2013, 78, 2792.

[32] (a) Kotnis, A. S. Tetrahedron Lett. 1991, 32, 3441. (b) Kotnis, A. S. Tetrahedron Lett. 1990, 31, 481.

[33] Yadav, J. S.; Reddy, B. V. S.; Sabitha, G.; Reddy, G. S. K. K. Synthesis 2000, 1532.

[34] (a) Cruickshank, F. R.; Benson, S. W. J. Phys. Chem. 1969, 73, 733. (b) Cotton, F. A.; Wilkinson, G. Advanced Inorganic Chemistry, 5th ed., Wiley, Toronto, 1988.

[35] (a) Prout, C. K.; Wright, J. D. Angew. Chem., Int. Ed. Engl. 1968, 7, 659 .

(b) Bent, H. A. Chem. Rev. 1968, 68, 587.

[36] Ramalinga, K.; Vijayalakshmi, P.; Kaimal, T. N. B. Tetrahedron Lett. 2002, 43, 879.

[37] Das, S.; Borah, R.; Devi, R. R.; Thakur, A. J. Synlett 2008, 2741.

[38] Vaino, A. R.; Szarek, W. A. Chem. Commun. 1996, 2351.

[39] (a) Wahlstrom, J. L.; Ronald, R. C. J. Org. Chem. 1998, 63, 6021.

(b) Donohoe, T. J.; Harris, R. M.; Williams, O.; Hargaden, G. C. J. Am. Chem. Soc. 2009, 131, 12854.

[40] Jereb, M.; Vražič, D.; Zupan, M. Tetrahedron 2011, 67, 1355.

[41] (a) Lin, X.-F.; Cui, S.-L.; Wang, Y-G. Tetrahedron Lett. 2006, 47, 3127.

(b) Lin, C.; Fang, H.; Tu, Z.; Liu, J.-T.; Yao, C.-F. J. Org. Chem. 2006, 71,6588 .

(c) Li, X.; Mao, Z.; Wang, Y.; Chen, W.; Lin, X. Tetrahedron 2011, 67,3858 .

(d) Chu, C.-M.; Gao, S.; Sastry, M. N. V.; Yao, C.-F. Tetrahedron Lett. 2005, 46, 4971.

(e) Lingam, Y.; Rao, D. M.; Bhowmik, D. R.; Santu, P. S.; Rao, K. R.; Slam, A. I. Tetrahedron Lett. 2007, 48, 7243.

(f) Cox, E. D.; Cook, J. M. Chem. Rev. 1995, 95, 1797.
[42] Lin, X.-F.; Cui, S.-L.; Wang, Y.-G. Teteahedron Lett. 2006, 47, 3127.

[43] Ji, S. J.; Wang, S. Y.; Zhang, Y.; Loh, T. P. Tetrahedron 2004, 60, 2051.

[44] Mphahlele, M. J. Molecules 2009, 14, 4814.

[45] Bougault, M. J. C. R. Hebd Seances Acad. Sci. 1904, 139, 864.

[46] Brown, R. S.; Nagorski, R. W.; Bennet, A. J.; McClung, R. E. D.; Aarts, G. H. M.; Klobukowski, M.; McDonald, R.; Santarsiero, B. D. J. Am. Chem. Soc. 1994, 116, 2448.

[47] (a) Dowle, M. D.; Davies, D. I. Chem. Soc. Rev. 1979, 8, 171.

(b) Cardillo, G.; Orena, M. Tetrahedron 1990, 46, 3321.

(c) Robin, S.; Rousseau, G. Tetrahedron 1998, 54, 13681.

(d) van Tamelen, E. E.; Shamma, M. J. Am. Chem. Soc. 1954, 76, 2315.

(e) Bernett, G.; Doi. J. T.; Musker, W. K. J. Org. Chem. 1985, 50, 2048.

(f) Cuzzupe, A. N.; Di Florio, R.; Rizzacasa, M. A. J. Org. Chem. 2002, 67, 4392 .

[48] (a) Denmark, S. E.; Burk, M. T. Proc. Natl. Acad. Sci. U. S. A. 2010, 107, 20655.

(b) Veitch, G. E.; Jacobsen, E. N. Angew. Chem., Int. Ed. 2010, 49, 7332.

[49] (a) Blot, V.; Reboul, V.; Metzner, P. J. Org. Chem. 2004, 69, 1196. (b) Murai, T.; Niwa, H.; Kimura, T.; Shibahara, F. Chem. Lett. 2004, 33, 508 .

(c) Barks, J. M.; Knight, D. W.; Seaman, C. J.; Weingarten, G. G. Tetrahedron Lett. 1994, 35, 7259.

(d) Jones, A. D.; Knight, D. W. Chem. Commun. 1996, 915.

[50] Fu, C.; Ma, S. Eur. J. Org. Chem. 2005, 3942.

[51] (a) Baldwin, J. E. J. Chem. Soc., Chem. Commun. 1976, 734. (b) Baldwin, J. E. J. Chem. Soc., Chem. Commun. 1976, 736. (c) Baldwin, J. E. J. Chem. Soc., Chem. Commun. 1976, 738.

[52] Arcadi, A.; Cacchi, S.; Fabrizi, G.; Marinelli, F.; Moro, L. Synlett 1999, 1432.

[53] Arcadi, A.; Cacchi, S.; Giuseppe, S. D.; Fabrizi, G.; Marinelli, F. Org. Lett. 2002, 4, 2409.

[54] (a) Bew, S. P.; Knight, D. W. J. Chem. Soc., Chem. Commun. 1996, 1007.

(b) El-Tach, G. M.; Evans, A. B.; Knight, D. W.; Jones, S. Tetrahedron Lett. 2001, 42, 5945.

[55] Eicher, T.; Hauptmann, S.; Speicher, A. The Chemistry of Heterocycles, 2nd ed., Wiley-VCH, Weinheim, 2003, pp. 52 62 .

[56] Liu, Y.; Zhou, S. Org. Lett. 2005, 7, 4609.

[57] Waldo, J. P.; Larock, R. C. Org. Lett. 2005, 7, 5203.

[58] Peng, A. Y.; Ding, Y. X. Org. Lett. 2004, 6, 1119.

[59] Amjad, M.; Knight, D. W. Tetrahedron Lett. 2004, 45, 539.

[60] Yao, T.; Larock, R. C. J. Org. Chem. 2005, 70, 1432.

[61] Foot, O. F.; Knight, D. W.; Cheng, A.; Low, L.; Li, Y.-F. Tetrahedron Lett. 2007, 48, 647.

[62] Hessian, K. O.; Flynn, B. L. Org. Lett. 2006, 8, 243.

[63] Ali, S.; Zhu, H.; Xia, X. F.; Ji, K. G.; Yang, Y. F.; Song, X. R.; Liang, Y. M. Org. Lett. 2011, 13, 2598.

[64] Huang, Q.; Hunter, J. A.; Larock, R. C. J. Org. Chem. 2002, 67, 3437.

[65] (a) Fischer, D.; Tomeba, H.; Pahadi, N. K.; Patil, N. T.; Huo, Z.; Yamamoto, Y. J. Am. Chem. Soc. 2008, 130, 15720.

(b) Huo, Z.; Gridnev, I. D.; Yamamoto, Y. J. Org. Chem. 2010, 75, 1266.

[66] Huo, Z.; Yamamoto, Y. Tetrahedron Lett. 2009, 50, 3651.

[67] (a) Larock, R. C.; Yue, D. Tetrahedron Lett. 2001, 42, 6011.

(b) Hessian, K. O.; Flynn, B. L. Org. Lett. 2003, 5, 4377.

(c) Ren, X.-F.; Turos, E. Tetrahedron Lett. 1993, 34, 1575 
[68] Kitagawa, O.; Inoue, T.; Hirano, K.; Taguchi, T. J. Org. Chem. 1993, 58, 3106.

[69] Khan, Z. A.; Wirth, T. Org. Lett. 2009, 11, 229.

[70] Zhang, X.; Sarkar, S.; Larock, R. C. J. Org. Chem. 2006, 71, 236.

[71] Fei, N.; Hou, Q.; Wang, S.; Wang, H.; Yao, Z. J. Org. Biomol. Chem. 2010, 8, 4096.

[72] Verma, A. K.; Shukla, S. P.; Singh, J.; Rustagi, V. J. Org. Chem. 2011, 76, 5670 .

[73] Mehta, S.; Waldo, J. P.; Larock, R. C. J. Org. Chem. 2009, 74, 1141.

[74] (a) Song, H.; Liu, Y.; Wang, Q. Org. Lett. 2013, 15, 3274.

(b) Chen, C.-C.; Yang, S.-C.; Wu, M.-J. J. Org. Chem. 2011, 76, 10269.

(c) Ferrara, G.; Jin, T. N.; Akhtaruzzaman, M.; Islamc, A.; Han, L. Y.; Jiang, H.; Yamamoto, Y. Tetrahedron Lett. 2012, 53, 1946.

(d) Xie, Y.-X.; Yan, Z.-Y.; Qian, B.; Deng, W.-Y.; Wang, D.-Z.; Wu, L.-Y.; Liu, X.-Y.; Liang, Y.-M. Chem. Commun. 2009, 5451.

[75] Batchu, H.; Bhattacharyya, S.; Batra, S. Org. Lett. 2012, 14, 6330.

[76] Bharathiraja, G.; Sakthivel, S.; Sengoden, M.; Punniyamurthy, T. Org. Lett. 2013, 15, 4996.

[77] Gao, W.-C.; Wang, R.-L.; Zhang, C. Org. Biomol. Chem. 2013, 11, 7123.

[78] Gao, Q.-H.; Fei, Z.; Zhu, Y.-P.; Lian, M.; Jia, F.-C.; Liu, M.-C.; She, N.-F.; Wu, A.-X. Tetrahedron 2013, 69, 22.

[79] (a) Huang, H. W.; Ji, X. C.; Wu, W. Q.; Jiang, H. F. Adv. Synth. Catal. 2013, 355, 170.

(b) Zhao, J.; Huang, H.; Wu, W.; Chen, H.; Jiang, H. Org. Lett. 2013, 15, 2604.

[80] Dhineshkumar, J.; Lamani, M.; Alagiri, K.; Prabhu, K. R. Org. Lett. 2013, 15, 1092.

[81] (a) Wan, C.; Zhang, J.; Wang, S.; Fan, J.; Wang, Z. Org. Lett. 2010, 12, 2338 .

(b) Wan, C.; Gao, L.; Wang, Q.; Zhang, J.; Wang, Z. Org. Lett. 2010, 12, 3902.

[82] (a) Zhang, J. T.; Zhu, D. P.; Yu, C. M.; Wan, C. F.; Wang, Z. Y. Org. Lett. 2010, 12, 2841. (b) Yan, Y. Z.; Wang, Z. Y. Chem. Commun. 2011, 47, 9513.

[83] Wang, Q.; Wan, C.; Gu, Y.; Zhang, J.; Gao, L.; Wang, Z. Green Chem. 2011, 13, 578.

[84] Yan, Y.; Wang, Z. Chem. Commun. 2011, 47, 9513.

[85] Xu, K.; Hu, Y.; Zhang, S.; Zha, Z.; Wang, Z. Chem. Eur. J. 2012, 18, 9793.

[86] Xiao, F.; Chen, H.; Xie, H.; Chen, S.; Yang, L.; Deng, G.-J. Org. Lett. 2014, 16, 50.

[87] Nobuta, T.; Tada, N.; Fujiya, A.; Kariya, A.; Miura, T.; Itoh, A. Org. Lett. 2013, 15, 574.

[88] Zhu, Y.-P.; Liu, M.-C.; Cai, Q.; Jia, F.-C.; Wu, A.-X. Chem. Eur. J. 2013, 19, 10132.

[89] He, Z.; Liu, W.; Li, Z. Chem. Asian J. 2011, 6, 1340.

[90] Francisco, C. G.; Freire, R.; Herrera, A. J.; Peréz-Martín, I.; Suárez, E. Org. Lett. 2002, 4, 1959.

[91] (a) Fan, R.; Pu, D.; Wen, F.; Wu, J. J. Org. Chem. 2007, 72, 8994.

(b) Francisco, C. G.; Herrera, A. J.; Suárez, E. J. Org. Chem. 2003, $68,1012$.

(c) Katohgi, M.; Togo, H.; Yamaguchi, K.; Yokoyama, M. Tetrahedron 1999, 55, 14885.

(d) Koag, M.; Lee, S. Org. Lett. 2011, 13, 4766.

[92] (a) Donohoe, T. J.; Kabeshov, M. A.; Rathi, A. H.; Smith, I. E. D. Synlett 2010, 2956.

(b) Gao, M.; Yang, Y.; Wu, Y.-D.; Deng, C.; Cao, L.-P.; Meng, X.-G.; Wu, A.-X. Org. Lett. 2010, 12, 1856.

[93] (a) Wang, Z.; Yin, G.; Qin, J.; Gao, M.; Cao, L.; Wu, A. Synthesis 2008, 3675.

(b) Gao, M.; Yin, G.; Wang, Z.; Wu, Y.; Guo, C.; Pan, Y.; Wu, A. Tetrahedron 2009, 65, 6047.

(c) Xue, W.-J; Guo, Y.-Q.; Gao, F.-F.; Li, H.-Z.; Wu, A.-X. Org. Lett. 2013, 15, 890.

(d) Zhu, Y.-P.; Gao, Q.-H.; Lian, M.; Yuan, J.-J.; Liu, M.-C.; Zhao, Q.; Yang, Y.; Wu, A.-X. Chem. Commun. 2011, 47, 12700.

[94] Xu, W.; Kloeckner, U.; Nachtsheim, B. J. J. Org. Chem. 2013, 78, 6065. 